\title{
La desigualdad del ingreso desde una perspectiva estructural
}


\section{Introducción}

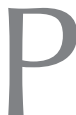

ara comenzar la comprensión de este tema, es preciso partir del hecho que las extremas desigualdades en El Salvador, primordialmente en términos de ingreso, poseen profundas raíces históricas (Banco Mundial 2004: 7). Se pretende sentar las bases de un estudio que buscaría establecer que la desigualdad en esta región no puede explicarse 
únicamente, como algunos han sostenido, como resultado de políticas impuestas al interior de los países latinoamericanos, Ilámeseles industrialización por sustitución de importaciones, Consenso de Washington, etc. Sin embargo, es claro que la historia condiciona, pero no determina para siempre el futuro de las naciones: "Siempre hay espacio para los cambios de rumbo, incluso para la erradicación de las injustas desigualdades en América Latina" (Marc Bou i Noversa 2005:1).

Para perseguir el objetivo de la presente investigación, es importante abordar la teoría económica que ha venido marcando tendencia en materia de distribución del ingreso, buscando encontrar las verdaderas raíces de esta desigualdad, contrariamente a las manifestaciones que históricamente se han buscado mitigar, como la baja y dispareja calidad educacional; los altos niveles de concentración de la tierra y otros activos productivos; el acceso desigual a mercados financieros $y$ laborales; la mala redistribución a través del Estado (considerada débil, dada la regresividad de la política fiscal aplicada desde hace varias décadas) entre otros.

$Y$ es que la problemática generada por la desigualdad ha representado un impacto negativo en el crecimiento económico. Al mismo tiempo, la existencia de desarticulación en el aparato productivo provoca esta desigualdad y también exclusión. Además, dicha situación se ha visto fortalecida por el modelo económico vigente y del cual pretendemos analizar su dinámica y considerar si su eje dinámico tiene alguna incidencia en la persistencia de este fenómeno.

Dicho lo anterior, es indiscutible señalar que el presente estudio pretende centrar su atención en que existen obstáculos estructurales que imposibilitan la tendencia hacia un equilibrio, y que esto a su vez puede deducir que el problema de exclusión y desigualdad está siendo provocada por una inadecuada estructura productiva que no ha sabido distribuir los beneficios del crecimiento económico ni ha podido frenar la concentración de pocos y la pauperización de las mayorías, afectando así a la distribución de las rentas entre los agentes económicos.

Por todos estos motivos, se ha concretado que el objetivo primordial de este estudio será el establecer la relación entre la estructura productiva y la desigualdad de los ingresos en El Salvador, en el periodo de 1986 al 2006 desde un enfoque estructural.

\section{Revisión de las distintas medidas de desigualdad en El Salvador}

Dara medir la distribución de los ingresos, es necesario recurrir a indicadores que cuantifiquen la situación actual de este fenómeno en la economía sal- 
vadoreña. Asimismo, estos deben guardar relación con los dos componentes a examinar en el presente apartado: primero, la tipificación y análisis de las familias salvadoreñas de acuerdo a su nivel de ingresos, y segundo, la medición del bienestar de los individuos a través de una medida de desigualdad, Ilámese índice de Gini, Theil, etc.

Ahora bien, es necesario que para preparar el análisis mencionado, mostrar la evolución de la economía salvadoreña en las últimas décadas, a través del estudio de su desarrollo, para luego ahondar en su evolución desde la perspectiva de los niveles de ingresos.

Posteriormente, se hace un recuento de las principales ventajas, críticas y limitaciones que se les atribuyen a los índices de desigualdad de la economía salvadoreña en los último años, para ello, el estudio permitió escoger como herramienta de cálculo principal la Encuesta de Hogares de Propósitos Múltiples, esto debido a que es el instrumento que mejor representa las ventajas y limitantes de las familias en El Salvador. Es gracias a ella que se construyen y se comparan los diversos índices de desigualdad.

\subsection{Breve análisis del crecimiento económico de la economía salva- doreña}

En las últimas cuatro décadas, El Salvador ha pasado de un modelo económico de industrialización por sustitución de importaciones, en el que el Estado jugaba un rol determinante en tanto orientador de la actividad económica, hacia otro, aún vigente, orientado por el mercado.

\section{Cuadro $N^{\circ} 1.1$}

\section{Crecimiento económico por décadas de El Salvador (variaciones porcentuales )}

\begin{tabular}{|c|c|c|c|c|}
\hline Década & PIB & Sector Agrícola & Sector Manufacturero & Sector Servicios \\
\hline 1960 & 5.9 & 3.6 & 8.6 & 2.9 \\
\hline 1970 & $4(5.0)$ & $3.3(3.6)$ & $2.9(4.5)$ & $5.3(6.1)$ \\
\hline 1980 & -0.9 & -2.3 & -1.5 & -0.7 \\
\hline 1990 & 5.2 & 1.5 & 4.9 & 4.8 \\
\hline 2000-2006 & 2.6 & 1.5 & 2.7 & 3.1 \\
\hline PROMEDIO & 3.36 & 1.52 & 3.52 & 3.1 \\
\hline
\end{tabular}

Fuente: Elaboración propia con datos de la CEPAL y el Banco Central de Reserva de El Salvador. * Las cifras entre paréntesis representan el crecimiento económico de 1970 a 1978. Las demás representan el crecimiento obtenido en toda la década (Es decir, de 1970 a 1980).

Un breve vistazo al cuadro 1.1 nos explica que en la década de 1960 fue el momento en la historia de los últimos 48 años en que El Salvador tuvo las mayores tasas de crecimiento económico. 
En la transición hacia la década de los setenta el crecimiento comienza a decaer progresivamente, obteniendo tasas moderadas y menores al 5\%, debido principalmente por el debilitamiento del Mercado Común Centroamericano (MCCA), la pérdida de capacidad de continuar impulsando la sustitución de importaciones y las vísperas de un conflicto armado. Aunque la década de los setenta, estudiada como un todo, muestra un dinamismo cercano al $4 \%$, el verdadero crecimiento de la década fue perturbado por la incertidumbre del conflicto armado acontecida en 1979, al ser eliminado dicho año, el crecimiento asciende al 5\%. Aun así, el modelo predominante de la época ya mostraba señales de agotamiento.

Durante los años ochenta, en medio de una guerra civil, el PIB retrocedió en su crecimiento como nunca, con una tasa promedio de $-0.9 \%$; todos los sectores económicos fueron afectados, especialmente la agricultura, que fue dañada por el combate armado en las zonas rurales del país.

Es primordial examinar las razones de porqué el crecimiento de los años recientes no ha logrado alcanzar el dinamismo esperado, cuando hoy, los organismos multilaterales (FMI, BM, BID) enaltecen a la economía salvadoreña, afirmando que es una de las que más ha obedecido lo propuesto por el Consenso de Washington, ya que posee una de las estructuras de mercado "más abiertas" de la región, precios que obedecen a las fuerzas del mercado, parte de la infraestructura está ahora en manos privadas, etc.

\section{Cuadro No 1.2 \\ Producto Interno Bruto total y variación anual (A precios constantes de 1990, Cifras en Millones de Dólares)}

\begin{tabular}{|c|c|c|}
\hline AÑo & PIB & VARIACIÓN \% \\
\hline 1993 & 5741.8 & 7.4 \\
\hline 1994 & 6089.3 & 6.1 \\
\hline 1995 & 6478.7 & 6.4 \\
\hline 1996 & 6589.2 & 1.7 \\
\hline 1997 & $6,869.0$ & 4.2 \\
\hline 1998 & $7,126.5$ & 3.7 \\
\hline 1999 & 7372.4 & 3.4 \\
\hline 2000 & $7,531.0$ & 2.2 \\
\hline 2001 & $7,659.7$ & 1.7 \\
\hline 2002 & $7,839.0$ & 2.3 \\
\hline 2003 & 8019.3 & 2.3 \\
\hline 2004 & 8167.7 & 1.9 \\
\hline 2005 & $8,419.7$ & 3.1 \\
\hline 2006 & $8,772.0$ & 4.2 \\
\hline
\end{tabular}

Fuente: Banco Central de Reserva 
El cuadro $\mathrm{N}^{\circ} 1.2$ muestra la evolución del crecimiento de la economía salvadoreña desde la culminación de la guerra a la fecha. En primer lugar, es necesario señalar que a partir del año 1996 existió una desaceleración de la economía y no por algún evento circunstancial que pudo corregirse, sino que ese bajo rendimiento se mantuvo aproximadamente una década, al punto de culminar el primer quinquenio del siglo XXI con niveles de bajo crecimiento.

Pareciera ser que, con la entrada del milenio. el modelo tiene encerrado cierto potencial de crecimiento superior que el mostrado a la fecha, ya que se refleja una racha de crecimiento de la actividad económica a partir del año 2005, causada principalmente por la exportación de productos no tradicionales, el incremento de los precios del café, el incremento en empresas de servicios (principalmente en el sector turismo) y la reactivación del agro. (Chalabi, N. 2008:1).

Si bien, este cambio del ritmo de crecimiento ha mejorado las expectativas económicas respecto a la década anterior, resulta ser insuficiente para superar el promedio de los países de la región y mucho menos las tasas de crecimiento que mantenía el país en los años de la ISI. Es Ilegado aquí, donde en definitiva se hace necesario proponer un plan alternativo de desarrollo a largo plazo o modificaciones inmediatas al modelo vigente; ya que, según las perspectivas de crecimiento mundial otorgadas por el Fondo Monetario Internacional, la desaceleración de la economía estadounidense es inminente $y$ países como El Salvador, se verán disminuidos en sus perspectivas de crecimiento a futuro.

\subsubsection{Producto Interno Bruto por habitante}

El PIB per cápita se utiliza frecuentemente como indicador del nivel medio de vida de una economía. Pero, para que la condición media de la población mejore, el nivel de crecimiento del PIB per cápita debe superar la variación del crecimiento poblacional dentro de un país. Observando el caso de El Salvador en el año 1997, la variación del PIB por habitante a precios constantes de 1990 fue igual al incremento de la población según las proyecciones de la Dirección General de Estadísticas y Censos (DIGESTYC). 


\section{Cuadro $N^{\circ} 1.3$}

Producto Interno Bruto por habitante a precios constantes de 1990

\begin{tabular}{|c|c|c|c|c|c|}
\hline AÑO & $\begin{array}{c}\text { PIB (En } \\
\text { millones de } \\
\text { dólares) }\end{array}$ & $\begin{array}{c}\text { POBLACIÓN } \\
\text { (En miles de } \\
\text { habitantes) }\end{array}$ & $\begin{array}{c}\text { VAR. } \\
\text { ANUAL } \\
\%\end{array}$ & $\begin{array}{c}\text { PIB POR } \\
\text { HABITANTE }\end{array}$ & $\begin{array}{c}\text { VAR. } \\
\text { ANUAL } \\
\%\end{array}$ \\
\hline 1997 & $6,869.0$ & $5,908.5$ & 2.1 & $1,162.6$ & 2.1 \\
\hline 1998 & $7,126.5$ & $6,031.3$ & 2.1 & $1,181.6$ & 1.6 \\
\hline 1999 & $7,372.4$ & $6,154.3$ & 2.0 & $1,197.9$ & 1.4 \\
\hline 2000 & $7,531.0$ & $6,276.0$ & 2.0 & $1,200.0$ & 0.2 \\
\hline 2001 & $7,659.7$ & $6,396.9$ & 1.9 & $1,197.4$ & -0.2 \\
\hline 2002 & $7,839.0$ & $6,517.8$ & 1.9 & $1,202.7$ & 0.4 \\
\hline 2003 & $8,019.3$ & $6,638.2$ & 1.8 & $1,208.1$ & 0.4 \\
\hline 2004 & $8,167.7$ & $6,864.1$ & 3.4 & $1,208.7$ & 0.0 \\
\hline 2005 & $8,419.7$ & $6,864.1$ & 0.0 & $1,224.7$ & 1.3 \\
\hline 2006 & $8,772.0$ & $6,980.3$ & 1.7 & $1,254.8$ & 2.5 \\
\hline
\end{tabular}

Fuente: Elaboración propia utilizando datos del BCR.

En el cuadro $\mathrm{N}^{\circ} 2.3$ se presenta el PIB per cápita, su variación porcentual y la población con su respectiva tasa de crecimiento anual. A partir de 1998, el PIB per cápita reportó un crecimiento menor que la variación anual de la población; lo que significa que el leve crecimiento económico per cápita no tiene efecto alguno en el nivel de vida la población, ya que esta se incrementó a un ritmo superior que el primero.

\subsubsection{La distribución del ingreso en El Salvador}

América Latina se ha caracterizado históricamente por demostrar una estructura de distribución del ingreso sumamente concentrada. El Salvador, por su parte, no ha estado ajeno a esta realidad, tal y como se representa en el cuadro $\mathrm{N}^{0} 1.4$. Así notamos que desde 1961, por ejemplo, el 20\% más pobre de la población recibía el $6 \%$ del ingreso nacional y por otro extremo, el $20 \%$ más rico capturaba el $61 \%$ del ingreso nacional. Luego, en la década de los setenta, la estructura distributiva se caracterizó por profundizar aún más las desigualdades en el ingreso, a tal punto que mientras el $20 \%$ más pobre redujo su participación en los ingresos totales a $3 \%$, el 20\% más rico incrementó su participación en un $66 \%$. Estas crecientes desigualdades constituyeron un factor que incidió notablemente en la alta polarización social y que tuvo como resultado, el estallido del conflicto armado durante los ochenta.

A comienzos de la década de los noventa, luego del conflicto armado la situación había mejorado ligeramente, ya que el quintil más pobre de la población incrementó su participación en los ingresos totales a 3\%, mientras que el quintil de la población de más altos in- 
gresos disminuyó su participación $54 \%$. No obstante, en el período siguiente se volvió a deteriorar, hasta que en el 2002 el quintil más pobre disminuyó su participación a 2.4\% del total de ingresos y el más rico la aumentó a 58.3\%.

\section{Cuadro No 1.4}

Distribución del ingreso por hogares (varios años)

\begin{tabular}{|c|c|c|}
\hline \multirow{2}{*}{ Año } & \multicolumn{2}{c|}{ Estructura de distribución del ingreso. } \\
$\mathbf{2 0 \%}$ más pobre & $\mathbf{2 0 \%}$ más rico \\
\hline 1961 & $6 \%$ & $61 \%$ \\
\hline 1969 & $4 \%$ & $51 \%$ \\
\hline 1979 & $2 \%$ & $66 \%$ \\
\hline 1992 & $3.2 \%$ & $54.5 \%$ \\
\hline 2002 & $2.4 \%$ & $58.3 \%$ \\
\hline 2005 & $4.1 \%$ & $54 \%$ \\
\hline 2006 & $4.5 \%$ & $51 \%$ \\
\hline
\end{tabular}

Fuente: Elaboración propia según CEPAL y EHPM (Varios Años)

No obstante, observamos que existen signos de recuperación en la distribución de los ingresos a partir del año 2005 y que nos muestra que para el año 2006 el quintil de más bajos ingresos obtuvo una participación de un $4.5 \%$ y que por el contrario, el quintil más rico ha visto reducida su proporción de ingresos a un $51 \%$.

Sin embargo, habría que señalar que estos signos de "recuperación", están auspiciados, en gran medida, por el auge en las remesas familiares y no por el fortalecimiento del aparato productivo. De no ser por las remesas, El Salvador sería no solo un país con más población en situación de pobreza, sino también un país bastante más desigual.

\subsubsection{Evolución de la distribución del ingreso entre 1997-2006}

En esta parte se examina cómo últimamente ha evolucionado el ingreso de los hogares de acuerdo a la Encuesta de Hogares de Propósitos Múltiples de El Salvador, para lo cual centraremos nuestra atención en cuatro años: 1997, 2000, 2003 y 2006.

En el periodo 1997-2006, el ritmo de crecimiento del ingreso no ha sido uniforme (ver cuadro $\mathrm{N}^{\circ}$ 2.5). Al analizar, por ejemplo, el avance del año 2000 respecto a 1997, se observa que los deciles que poseen los ingresos más bajos presentaron una significativa reducción en sus economías familiares. De manera contraria, del tercer decil al décimo decil todas las variaciones fueron positivas, generando así incrementos de $7.86 \%$ hasta $20.53 \%$ para el decil de mayor nivel de ingreso.

A continuación, los gráficos $2.1,2.2$ y 2.3 corresponden a las comparaciones porcentuales 
de los ingresos percibidos según cada decil de hogares para los años:2000/1997, 2003/2000 y 2006/2003 respectivamente.

\section{Gráfico No 2.1}

\section{Evolución del ingreso por decil 2000/1997 (En porcentajes)}

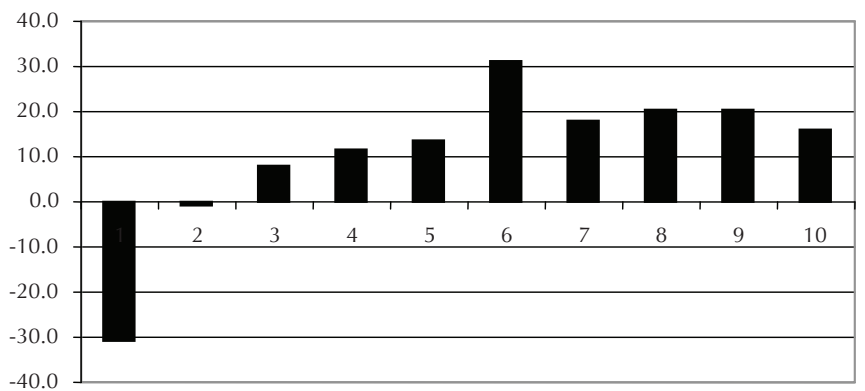

Fuente: Elaboración propia según EHPM 1997/2000.

El gráfico $\mathrm{N}^{\circ} 2.2$ explica la evolución en el ingreso familiar por deciles, entre el año 1997 y 2000. Se puede observar una notoria concentración del ingreso, dado el claro incremento de los ingresos para todos los deciles menos para el primero y el segundo. Inversamente, de 2000 al 2003 (Gráfico No 2.3.) se expone una mejoría en la distribución del ingreso para los deciles menores más que los deciles más ricos, exceptuando el último decil, el cual aumentó su proporción en un aproximado del $4 \%$.

\section{Gráfico No 2.2}

Evoluión del ingreso por decil 2003/2000 (En porcentajes)



Fuente: Elaboración propia según EHPM 2000/2003.

Por último observamos una familiar en todos los deciles entre mejora en el desarrollo del ingreso los años 2003 y 2006 (ver gráfico 
$N^{0}$ 2.4.). Una posible explicación puede deberse al incremento en el gasto social de las familias más pobres por parte del Estado salvadoreño. Si bien esta comparación muestra un avance en términos absolutos, no es suficiente para sacar a las familias más pobres de la línea de pobreza, dada la tendencia actual de los precios de los alimentos.

Gráfico No 2.3

\section{Evolución del ingreso por decil 2006/2003 (En porcentajes)}

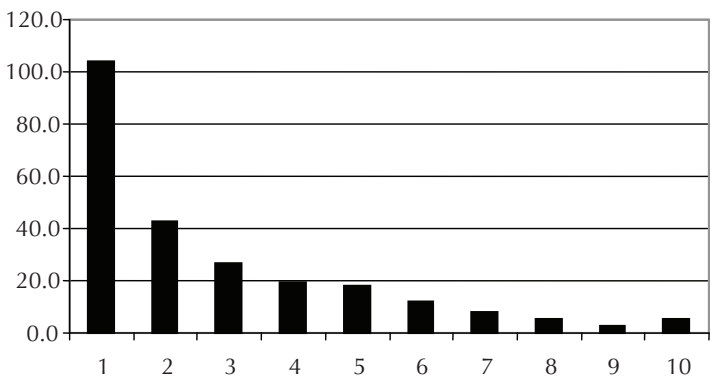

Fuente: Elaboración propia según EHPM 2003/2006.

Es notorio que a pesar de las últimas evidencias encontradas, la distribución del ingreso mantiene la misma tendencia, presentando una alta concentración en los hogares pertenecientes al decil de mayores ingresos del país. Sin embargo, la medición realizada para el año 2006 representa un punto de quiebre de esta situación. Observándose por primera vez en más de una década una mejoría en la distribución del ingreso en términos generales.

Esta mejora en la distribución del ingreso se puede ilustrar a través de los índices 10/10, 20/20, 10/40 y $20 / 80$, tal y como lo muestra el cuadro $\mathrm{N}^{\circ} 2.5$ El índice 10/10 se construye dividiendo la proporción del ingreso del $10 \%$ de los hogares más ricos por la proporción que recibe el $10 \%$ de los hogares de menores ingresos. Se aprecia que este se reduce desde un 42.2 en el 2003 a 21.7 en el 2006. Algo similar ocurre con el índice 20/20, en el que luego de oscilar en un 17.1 en el 2003, se redujo a un 11.2 en el 2006, el menor valor observado en los últimos años. 
Evolución de los índices 10/10, 20/20, 10/40 y 20/80, a partir de los ingresos (2000-2006)

\begin{tabular}{|lc|c|c|c|c|c|c|}
\multicolumn{1}{c}{ Año } \\
\multicolumn{1}{c}{ Índice } & $\mathbf{2 0 0 0}$ & $\mathbf{2 0 0 1}$ & $\mathbf{2 0 0 2}$ & $\mathbf{2 0 0 3}$ & $\mathbf{2 0 0 4}$ & $\mathbf{2 0 0 5}$ & $\mathbf{2 0 0 6}$ \\
Índice 10/10 & 36.9 & 25.9 & 50.3 & 42.0 & 26.6 & 26.6 & 21.7 \\
Índice 20/20 & 16.7 & 12.8 & 19.8 & 17.1 & 13.4 & 13.4 & 11.2 \\
\hline Índice 10/40 & 2.9 & 2.8 & 3.8 & 3.2 & 2.9 & 2.9 & 2.6 \\
Índice 20/80 & 1.1 & 1.1 & 1.3 & 1.1 & 1.1 & 1.1 & 1.0 \\
\hline
\end{tabular}

Fuente: Elaboración propia en base a EHPM (varios años).

No obstante los datos oficiales, es innegable que la distribución de los ingresos continúa siendo altamente desigual en el país, con el $20 \%$ de los hogares con mayores ingresos aún concentrando, para el 2006, más del $50 \%$ del total de los ingresos, en tanto que el $20 \%$ de los hogares más pobres reciben sólo el $4.5 \%$ de estos ingresos.

En la siguiente sección se trata de dar una explicación a la desigualdad de los ingresos por medio del análisis de la economía familiar.

\subsubsection{Análisis del ingreso familiar de los deciles más pobres}

Una de las manifestaciones más notorias de la desigualdad en la estructura socio-económica salvadoreña es el fenómeno de la pobreza. En El Salvador, ésta se mide (según la Dirección General de Estadísticas y Censos, DIGESTYC) a partir de la incapacidad de la economía de proveer los ingresos necesarios para suplir la demanda en el consumo básico de alimentos por parte de las familias. Este análisis se realiza a través de la Canasta Básica Alimen- taria (CBA) o de la Canasta Básica Ampliada (CA).

Aunque cabe considerar que dichas canastas no son los indicadores más aptos para la medición de la pobreza; primero, porque hacen referencia únicamente a bienes alimenticios, y en segundo lugar, porque el valor de la canasta de bienes que cubre el valor de la fuerza de trabajo varía con el tiempo, producto del aumento o disminución del poder adquisitivo, mientras que la CBA fijada por la DIGESTYC, no varía. Por lo tanto, no es un indicador que se ajusta apropiadamente a la evolución del nivel de vida de los trabajadores. (Escobar B, 2003:78)

Al examinar la evolución de la pobreza en los últimos años, se destaca que a pesar de la rápida reducción experimentada en los niveles de la pobreza y pobreza extrema en las zonas urbanas, existe una lenta disminución de estos niveles en las zonas rurales. Por lo que podemos distinguir que el lugar de residencia está siendo determinante en la distribución de los ingresos en El 
Salvador. Esto se evidencia además en que cerca del $85 \%$ de las familias ubicadas en el primer decil de ingresos residen en el área rural.

Las razones de esta peculiar disparidad en la evolución de la pobreza urbana y rural podría estar relacionada con el modelo de crecimiento, el cual se ha basado en el mayor dinamismo del sector terciario de la economía (sector comercial y de servicios) y que ha llevado al estancamiento del sector agropecuario, el cual ha experimentado una constante reducción en su importancia relativa, pasando de representar arriba del 18\% del PIB en 1985 , a $13.6 \%$ en $1995,11.8 \%$ en 2001 y $12.1 \%$ en el 2006 .

Siguiendo con el análisis, los resultados obtenidos en el cuadro 2.6 nos muestran que a pesar que los primeros deciles de ingresos han aumentado su peso relativo en la distribución de los ingresos de los últimos años, esta proporción aun mantiene a gran parte de las familias pertenecientes a los primeros dos deciles de ingresos bajo la línea de pobreza extrema. Para el caso del 2006, por ejemplo, la CBA rural fue de $\$ 99.00$ dólares nominales y en dicho año el ingreso promedio de una familia perteneciente al primer decíl de ingreso fue de $\$ 57.00$ $y$ del segundo, \$99.7. (EHPM 2006)

Si por otra parte, en lugar de la CBA, tomamos en cuenta la Canasta Básica Ampliada como un indicador más efectivo para medir el nivel de los ingresos de las familias, observamos que la cantidad de hogares que se encontrarían en una situación de pobreza relativa estaría incluida del decil I al IV, tanto para el caso del área urbana (los cuales poseen ingresos promedios de $\$ 89.1$ a $\$ 282.7$ y y al observar el valor de la CA en el cuadro 2.9 para el 2006, se obtiene un costo de 274 . Como para el área rural (Ingresos promedio que oscilan de $\$ 57.0$ a $\$ 164.6$ ) y con un valor de la CA de 198 para el 2006. Indica que las familias pierden cada vez más el poder para adquirir la CA.

\section{Cuadro No 2.6}

Costo de la canasta básica alimentaria rural y urbana

\begin{tabular}{|c|c|c|c|c|c|c|c|}
\hline Año & $\begin{array}{c}\text { CBA } \\
\text { Urbana }\end{array}$ & \multicolumn{1}{c}{$\begin{array}{c}\text { CBA } \\
\text { Rural }\end{array}$} & \multicolumn{2}{c}{$\begin{array}{c}\text { CA } \\
\text { Urbana }\end{array}$} & \multicolumn{2}{c|}{$\begin{array}{c}\text { CA } \\
\text { Rural }\end{array}$} & \multicolumn{2}{c|}{$\begin{array}{c}\text { IPC } \\
\text { Alimentos }\end{array}$} & \multicolumn{1}{c|}{$\begin{array}{c}\text { CBA } \\
\text { Urbana } \\
\text { Ajustada }\end{array}$} & $\begin{array}{c}\text { CBA } \\
\text { Rural } \\
\text { Ajustada }\end{array}$ \\
\hline 1997 & 143 & 111 & 286 & 222 & 158.3 & 170 & 119 \\
\hline 1998 & 141 & 103 & 282 & 206 & 170.4 & 183 & 128 \\
\hline 1999 & 136 & 98 & 272 & 196 & 159.46 & 171 & 120 \\
\hline 2000 & 128 & 99 & 256 & 198 & 163.44 & 176 & 123 \\
\hline 2001 & 129 & 98 & 258 & 196 & 167.85 & 181 & 126 \\
\hline 2002 & 127 & 93 & 254 & 186 & 169.2 & 182 & 127 \\
\hline 2003 & 126 & 90 & 252 & 180 & 176.4 & 190 & 133 \\
\hline 2004 & 130 & 96 & 260 & 192 & 188.6 & 203 & 142 \\
\hline 2005 & 137 & 98 & 274 & 196 & 197.6 & 212 & 148 \\
\hline 2006 & 137 & 99 & 274 & 198 & 207.99 & 223 & 156 \\
\hline 2007 & 146 & 110 & 292 & 220 & 221.43 & 237 & 166 \\
\hline
\end{tabular}

Fuente: Elaboración propia en base a PNUD y EHPM (varios años). 
Es válido mencionar que a pesar de la tendencia a la reducción paulatina de la población en pobreza extrema y pobreza relativa, aún existen profundas carencias en los deciles poblacionales de más bajos ingresos, lo que les impide cubrir por completo sus necesidades. Esto conduce a que gran parte de la población busque alternativas para satisfacer las necesidades básicas de sus familias, por ejemplo a través de la emigración o de actividades ilegales.

\subsection{Medidas de desigualdad en El Salvador}

Para hablar de medición de desigualdad es necesario conocer qué se entiende por dicho término. La medición de la desigualdad hace referencia a las diferencias que existen en la distribución de los ingresos de los hogares en una nación, entendiendo que dentro de este concepto se incluye el acceso que las personas tienen a los recursos sociales y económicos.

Si bien, la simple observación de los cuadros presentados en el apartado anterior nos da una idea de los grupos de la población más favorecidos, es útil medir la desigualdad empleando indicadores que resumen la situación general, tomando en cuenta los ingresos de toda la población.

Para ello, se ha considerado importante dar a conocer los criterios más utilizados en la medición de la desigualdad. Se desarrollaran a continuación el coeficiente de Gini, el índice de Theil y el índice de Atkinson.

\subsection{Criterios para medir la des- igualdad}

El índice de Gini y la curva de Lorenz son dos indicadores relacionados entre sí, que miden el grado de concentración de variables como el salario o la renta en un país. A continuación, veremos la conceptualización de cada uno de ellos.

\subsection{1. Índice de Gini para El Sal- vador}

A continuación, se presentan los porcentajes de la distribución de los ingresos en El Salvador, que percibe cada grupo para los años del 2002, 2004 y 2006 donde, el primer decil corresponde a aquellos que reciben la porción más pequeña de la renta fruto de la actividad económica, seguido de los deciles que perciben mayores niveles de renta, hasta llegar al décimo decil. 
Cuadro No 2.7

Porcentajes de la distribución de ingresos familiares anuales en EI Salvador por deciles.

\begin{tabular}{|c|c|c|c|}
\hline Decil & 2002 & 2004 & 2006 \\
\hline 1 er. decil & $0.77 \%$ & $1.35 \%$ & $1.61 \%$ \\
\hline $2^{\circ}$ decil & $2.03 \%$ & $2.59 \%$ & $2.93 \%$ \\
\hline $3^{\circ}$ decil & $3.18 \%$ & $3.67 \%$ & $4.02 \%$ \\
\hline $4^{\circ}$ decil & $4.32 \%$ & $4.70 \%$ & $5.05 \%$ \\
\hline $5^{\circ}$ decil & $5.60 \%$ & $5.93 \%$ & $6.32 \%$ \\
\hline $6^{\circ}$ decil & $7.17 \%$ & $7.42 \%$ & $7.71 \%$ \\
\hline $7^{\circ}$ decil & $9.16 \%$ & $9.37 \%$ & $9.45 \%$ \\
\hline $8^{\circ}$ decil & $12.08 \%$ & $12.18 \%$ & $11.95 \%$ \\
\hline $9^{\circ}$ decil & $16.87 \%$ & $16.81 \%$ & $16.00 \%$ \\
\hline $10^{\circ}$ decil & $38.82 \%$ & $35.98 \%$ & $34.95 \%$ \\
\hline
\end{tabular}

Fuente: Elaboración propia en base a datos de la División de Estadísticas Sociales y de EHPM, de varios años ${ }^{1}$.

Al analizar la estructura de la distribución de los ingresos presentada en el cuadro $\mathrm{N}^{\circ} 2.7$, para los años 2002, 2004 y 2006, es importante destacar la tendencia en la disminución de la proporción de ingresos que obtienen los deciles más ricos y el leve mejoramiento en la proporción de los ingresos en los deciles más pobres. Por ejemplo, los niveles de concentración del decil más rico durante el año 2002 significaron el $38.82 \%$ del ingreso nacional, mientras que para el 2004 un valor de $35.98 \%$ y el 2006 un $34.95 \%$. Al mismo tiempo observamos que el decil más pobre logro elevar su proporción de ingresos de $0.77 \%$ en el 2002 a $1.61 \%$ en el 2006. A pesar de estas modificaciones, aún se evidencian fuertes desequilibrios en la distribución de los ingresos. Asegurando además, que los sectores más afectados con la desigualdad son los sectores de más bajos ingresos; ya que, los porcentajes obtenidos de la distribución (incluso el $1.61 \%$ obtenido para el 2006) los coloca en situación de extrema pobreza.

A partir de la distribución de los ingresos presentados en el cuadro $N^{\circ} 10$, se procedió a la construcción del índice de Gini. Los resultados son presentados a continuación:

\section{Cuadro No 2.8}

\section{Índices de Gini para El Salvador 2002-2006²}

\begin{tabular}{|c|c|c|c|c|c|}
\hline $\begin{array}{c}\text { Índice de } \\
\text { Gini }\end{array}$ & $\mathbf{2 0 0 2}$ & $\mathbf{2 0 0 3}$ & $\mathbf{2 0 0 4}$ & $\mathbf{2 0 0 5}$ & $\mathbf{2 0 0 6}$ \\
\hline
\end{tabular}

Fuente: Elaboración propia en base a datos de EHPM, varios años 
Los datos del cuadro $N^{\circ} 2.8$ reflejan la evolución del índice de Gini a lo largo del período 20022006. Para el año 2002 se obtuvo un valor del 0.51, el cual se incrementó para el 2003 a 0.5403, convirtiéndose El Salvador, con este resultado, en el segundo país con mayores desigualdades en América Latina (PNUD, 2003). A partir del año 2004, la desigualdad se vio disminuida, logrando para ese año un Gini de 0.4693, para finalmente disminuir en el 2006 a 0.4457.

\section{Gráfico No 2.4}

Curva de Lorenz (distribución personal de la renta en El Salvador de los años 2002 y 2006.

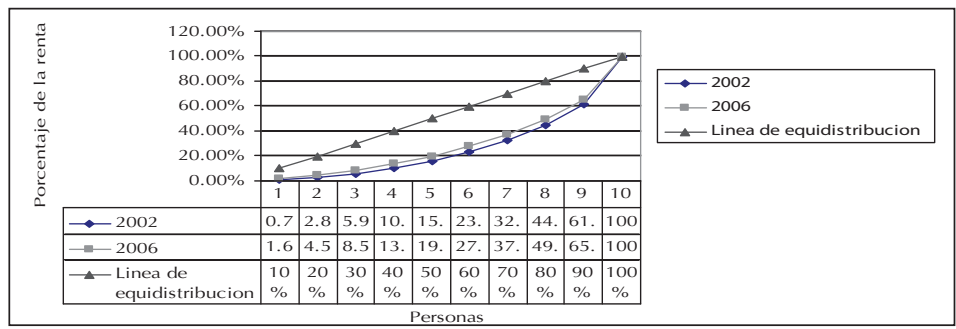

Fuente: Elaboración propia en base a datos de la División de Estadísticas Sociales Y de la EHPM 2002 y 2006.

Según el gráfico № 2.4, es posible decir que la desigualdad del año 2006 respecto al 2002 se ha visto disminuida, debido a que el área comprendida entre la curva de Lorenz y la de equidistribución sea menor para el año 2006. Pero si bien ha variado, se puede apreciar que el $10 \%$ más pobre aún no ha visto mejorado significativamente su nivel de ingresos, dado que para el 2002 percibió un porcentaje de la renta de $0.7 \%$ y para el 2006 ,
$1.6 \%$. Si se compara esta proporción de la población con el porcentaje de renta percibido por el $10 \%$ más rico, se notará que no habido cambios substanciales. Lo que habla de la realidad del país en cuanto a la existencia de desigualdad.

\subsection{2 Índice de Theil}

Para completar nuestro análisis, se ha calculado el índice de Theil para los años de 2002 al 2006, los cuales se presentan a continuación:

\section{Cuadro $\mathrm{N}^{\circ} 2.9^{3}$}

\section{Índice de Theil para El Salvador para los años del 2002-2006}

\begin{tabular}{|l|r|r|r|r|r|}
\hline Años & $\mathbf{2 0 0 2}$ & $\mathbf{2 0 0 3}$ & $\mathbf{2 0 0 4}$ & $\mathbf{2 0 0 5}$ & $\mathbf{2 0 0 6}$ \\
\hline $\mathrm{a}=1$ & $44.83 \%$ & $40.24 \%$ & $44.50 \%$ & $37.85 \%$ & $34.29 \%$ \\
\hline $\mathrm{a}=0$ & $50.72 \%$ & $45.83 \%$ & $42.90 \%$ & $39.98 \%$ & $35.21 \%$ \\
\hline
\end{tabular}

Fuente: Elaboración propia en base a datos EHPM, varios años 
Los datos nos reflejan con que para $\mathrm{a}=1$, los valores obtenidos capturan la sensibilidad en la cola alta de la distribución. Los resultados que se presentaron al calcular dicho índice es que para el año 2002, se tenía un valor de $44.83 \%$ y al 2006 un valor de $34.29 \%$, lo que implica que la desigualdad ha disminuido en $10.54 \%$.

En el caso en que el valor de $a=1$, los datos capturan la sensibilidad en la distribución en la cola baja de la distribución lo que nos indica que ha existido una reducción del 2002 con un valor de $50.72 \%$ al 2006 con $35.21 \%$ en la desigualdad.

Pero un fenómeno importante a resaltar es que los índices de Theil que se obtuvieron en la cola alta de la distribución, son menores que los presentados en la cola baja de la distribución. Lo que nos indica que las familias que perciben menores ingresos, en índice de Theil $\mathrm{a}=0$ son más sensibles a cualquier cambio existente en el nivel de ingreso, en comparación al impacto que existe en los niveles de ingreso mas elevados.

\subsection{3 Índice de Atkinson}

El índice de Atkinson se basa en una función de bienestar social. Atkinson además señala que toda medida de desigualdad implica un juicio de valor de carácter normativo. Para hacerlo explícito propone derivar medidas de desigualdad a partir de una función de bienestar concreta. El índice de igualdad de Atkinson se define entonces como el cociente entre el "ingreso igualmente distribuido" (ingreso medio que de ser compartido por todos los habitantes genera un nivel de bienestar semejante al actual) y el ingreso promedio de la economía. Esto es así, debido a que los juicios de valor sobre la desigualdad siempre tienen algún contenido normativo.

Por esa razón, algunos autores prefieren partir de la interpretación de la desigualdad como una pérdida potencial del bienestar colectivo, y es a partir de una función de bienestar social que se reflejan de forma explícita los juicios de valor acerca de la relación entre bienestar y desigualdad.

\section{Cuadro No 2.10}

Índice de Atkinson para El Salvador. 2002-2006 ${ }^{4}$

\begin{tabular}{|c|c|c|c|c|c|}
\hline Años & 2002 & 2003 & 2004 & 2005 & 2006 \\
\hline$\varepsilon=0.5$ & $21.32 \%$ & $19.40 \%$ & $17.84 \%$ ** & $15.50 \%$ & $16.08 \%$ \\
\hline$\varepsilon=1$ & $53.22 \%$ & $49.65 \%$ & $48.66 \%$ ** & $47.60 \%$ & $46.29 \%$ \\
\hline
\end{tabular}

Fuente: Elaboración propia en base a datos EHPM varios años

Los datos obtenidos del 2002 al 2006 con un grado de aversión a la desigualdad de $\varepsilon=0.5$ y de $\varepsilon=1$ se muestra que entre mayor sea este valor, más alta será la ponderación que reciben las observaciones que se ubican en el segmento inferior de la estructura de distribución. 
Pero además podemos ver que independientemente de la aversión a la desigualdad que se utilice, el índice de Atkinson se ha ido reduciendo. Pese a estos alentadores resultados, la desigualdad en $\mathrm{El}$ Salvador sigue caracterizándose por ser alta, ya que la magnitud de los cambios distributivos es muy pequeña.

\subsection{Análisis de la heterogeneidad estructural en El Salvador}

Es importante denotar la trascendencia que tiene la heterogeneidad estructural en la presente investigación, ya que es a través de su análisis que se busca dar a conocer los obstáculos estructurales que imposibilitan la tendencia hacia la equidad en materia distributiva, obstáculos que a su vez pueden ayudarnos a deducir que el problema de la exclusión y la desigualdad es provocado por una inadecuada estructura productiva.

En la noción de heterogeneidad estructural, se incluyen dos causas estructurales que son importantes a la hora de explicar los problemas de desarrollo en la región y su persistencia a lo largo del tiempo: la falta de vinculación entre sectores y los problemas de productividad que dificultan la inserción de la región a la economía mundial. Las dos son debilidades permanentes de El Salvador, pero que se han agravado como consecuencia de las políticas neoliberales.

Sin embargo, la heterogeneidad estructural no ha sido medida todavía por algún organismo gubernamental o privado salvadoreño, por lo que se hace necesario proponer algún método que logre calcularla.

Relación entre heterogeneidad estructural y el proceso de terciarización de la economía salvadoreña

La sociedad salvadoreña ha experimentando profundos cambios en su estrategia de crecimiento. El siguiente análisis muestra cómo la estructura productiva en El Salvador, durante los últimos años, ha

Gráfico № 2.5

Población ocupada por sector productivo, en porcentajes

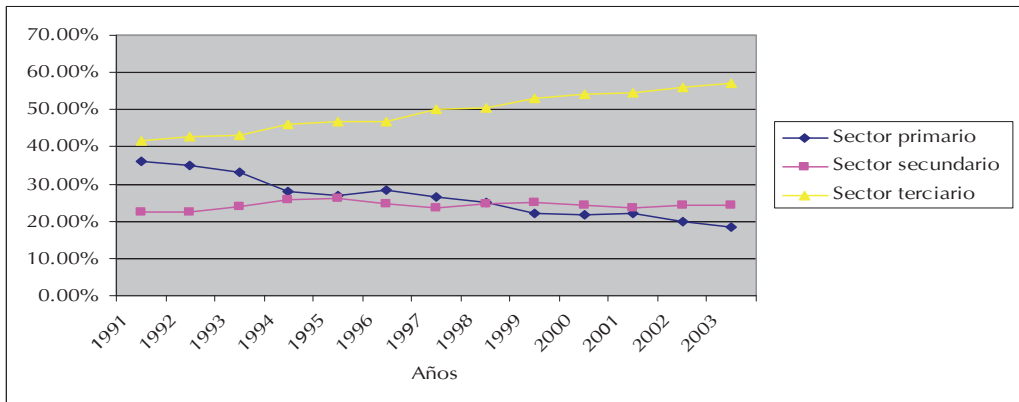

Fuente: Elaboración propia en base a datos de la EHPM, varios años. 
transitando desde un enfoque en el que se fortalecía a los sectores primario y secundario, a ser principalmente una economía sustentada en el comercio, servicios y finanzas, es decir una economía que ha desmontado el modelo agroexportador y que ha trasladado la mayor parte del capital hacia el sector terciario.

La tendencia a la tercerización ha traído aparejado un descuido en el sector primario de la economía, sector que, dada la participación histórica que mantenía en PIB, mantenía una extensa absorción laboral en las zonas principalmente agrícolas. La carencia de lugares de trabajo en el sector formal y el abandono de las tareas agrícolas, ha obligado a que muchas personas hayan creado sus propias ocupaciones laborales en actividades no regularizadas y caracterizadas por los bajos niveles de productividad y remuneración. Y es que las personas ocupadas en el sector informal cada vez son más, incluso representan cerca de la mitad de la población ocupada. Este sector contribuye a la reducción del desempleo y la pobreza en los hogares. No obstante, debido a la precariedad laboral, los problemas de desempleo y pobreza no están siendo resueltos, al contrario, se están agudizando, y con este proceso, las capacidades productivas de la mano de obra podrían estar siendo deterioradas irreversiblemente (Alfaro, Escoto y Sánchez, 2006:92).

Gráfico № 2.6

IVAE mensual por sectores de actividad económica (1990-2008)



Fuente: Elaboración propia en base a datos del BCR.

El gráfico № 2.6, muestra el resultado del dinamismo de la economía desde 1990, esto es medido a través del Índice de Volumen de la Actividad Económica (IVAE). Dicho indicador respalda lo antes dicho sobre el incremento en el ritmo de la actividad económica del sector terciario, frente al sector primario y secundario. Esto a su vez, genera una correspondencia con el sector informal, ya que al avanzar la economía a su proceso de terciarización, lo hace también el progreso 
tecnológico, lo cual genera un uso más intensivo en capital y por ende, una disminución en el uso de mano de obra.

\subsubsection{Propuesta para la medición de la heterogeneidad estructural a través de la dinámica ocupacional}

En el país no existe alguna medición de la heterogeneidad estructural, por lo que se hace necesario proponer un método para calcularla, es decir, construir un indicador que ayude a reflejar, aunque sea de forma aproximada, el nivel de este fenómeno.

Este indicador estará fundamentado en el impacto de la heterogeneidad estructural sobre la dinámica ocupacional principalmente. $Y$ es que la heterogeneidad estructural que presenta la economía salvadoreña coincide con la precarización del empleo y la insuficiente absorción laboral de la población dentro de los principales sectores económicos. Asimismo, se verá si a través del análisis de la actividad económica entre sectores (terciario vs. primario), el índice propuesto mejora.

Para la construcción de este indicador se hace referencia a dos componentes de la dinámica ocupacional salvadoreña (diagrama $\mathrm{N}^{\circ}$ 2.1). El primer componente a utilizar es una relación que refleja la brecha entre los ocupados plenos y los subempleados. Este último contiene dos modalidades de subempleados los visibles quienes son todas aquellas personas que estando ocupadas trabajan menos de 40 horas a las semana; y los subempleados invisibles quienes son personas que trabajan 40 horas semanales o más, obteniendo salarios menores al mínimo. El otro componente es un indicador que muestra la brecha entre los ocupados en el sector formal de la economía, con respecto a la cantidad de ocupados en el sector informal.

\section{Diagrama $\mathrm{N}^{\circ} 2.1$}

Componentes del indicador de heterogeneidad estructural

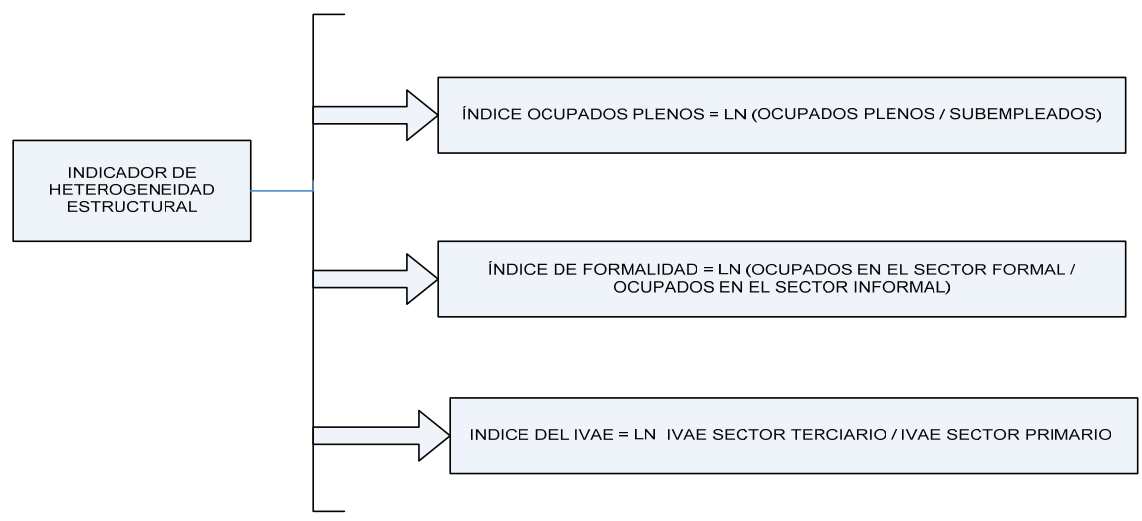

Fuente: Elaboración propia en base a datos del BCR. 
De este modo, se deducirá, a través de la dinámica ocupacional, qué nivel de eslabonamiento posee la economía entre sus principales sectores económicos, concluyendo que un mayor nivel de absorción laboral, reflejado ya sea, por el crecimiento de los ocupados plenos y/o ocupados formales, tenderá a la disminución de la heterogeneidad estructural, así como un crecimiento de las ocupados en el sector informal y/o el aumento de los subempleados sería producido por un incremento en el nivel de heterogeneidad estructural.

A continuación se brinda en detalle los indicadores que buscan analizar para la situación de heterogeneidad estructural en El Salvador.

\subsubsection{La heterogeneidad estructural medida a través del nivel de ocupación y subocupación}

$$
H E_{1}=L N\left(\frac{\text { OcupadosPlenos }}{\text { Subemepleados }}\right) \quad \begin{aligned}
& \begin{array}{l}
\mathrm{HE}>0 \\
\mathrm{HE}=0
\end{array} \\
& \mathrm{HE} \text { Si ocupados plenos es mayor que subempleados. }
\end{aligned}
$$

Este indicador nos muestra un cociente entre la población ocupada plenamente en la actividad económica, con respecto al número de trabajadores subempleados. El resultado de esta división nos describe cuál de los dos sectores está creciendo con mayor dinamismo. Si por ejemplo, el resultado (en logaritmo natural) nos muestra un número menor a 0 , significa que existe un mayor crecimiento de la población subempleada o una disminución del nivel de ocupación plena. Esto equivaldría a decir, que el aparato productivo no está absorbiendo plenamente a una mayor cantidad de personas dentro de las ramas del sistema económico y que, por lo tanto, estas personas recurren a subemplearse como medio efectivo para cubrir sus necesidades más elementales.

Si por el contrario, el resultado nos describe un numero mayor a 0 , puede deberse a que el crecimiento de la población subempleada es menor al crecimiento del número de ocupados plenos. El resultado nos explicaría una disminución del índice de heterogeneidad estructural $(\mathrm{HE})$, es decir que el aparato productivo está integrando eficazmente a la población dentro de la dinámica formal del sistema económico.

Los resultados de este indicador se presentan para un total de veintiún años (1986-2006), a continuación los datos obtenidos: 


\section{Cuadro $N^{\circ} 2.11$}

Indicador de heterogeneidad estructural (EH1) medido a través del nivel de ocupación y subocupación para El Salvador desde 1986-2006.

\begin{tabular}{|c|c|c|c|}
\hline \multicolumn{1}{|c|}{ Año } & Indicador HE & Año & Indicador HE \\
\hline $\mathbf{1 9 8 6}$ & -0.11 & $\mathbf{1 9 9 7}$ & 0.85 \\
\hline $\mathbf{1 9 8 7}$ & -0.16 & $\mathbf{1 9 9 8}$ & 0.77 \\
\hline $\mathbf{1 9 8 8}$ & -0.21 & $\mathbf{1 9 9 9}$ & 0.89 \\
\hline $\mathbf{1 9 8 9}$ & -0.10 & $\mathbf{2 0 0 0}$ & 1.02 \\
\hline $\mathbf{1 9 9 0}$ & 0.51 & $\mathbf{2 0 0 1}$ & 0.95 \\
\hline $\mathbf{1 9 9 1}$ & 0.55 & $\mathbf{2 0 0 2}$ & 0.86 \\
\hline $\mathbf{1 9 9 2}$ & 0.38 & $\mathbf{2 0 0 3}$ & 0.53 \\
\hline $\mathbf{1 9 9 3}$ & 0.69 & $\mathbf{2 0 0 4}$ & 0.60 \\
\hline $\mathbf{1 9 9 4}$ & 0.72 & $\mathbf{2 0 0 5}$ & 0.71 \\
\hline $\mathbf{1 9 9 5}$ & 0.77 & $\mathbf{2 0 0 6}$ & 0.52 \\
\hline $\mathbf{1 9 9 6}$ & 0.82 & & \\
\hline
\end{tabular}

Fuente: Elaboración propia en base a datos EHPM varios años.

Según los datos arrojados por el indicador de HE1 y que se presentan de forma gráfica a continuación, se observa que, en primer lugar existe, una tendencia (aunque irregular) a la disminución de la brecha entre los ocupados plenos y los subempleados, en el periodo comprendido de 1986 al 2000, lo que explicaría que el nivel de ocupados plenos aumentó con respecto al nivel de subempleados. Para el caso del período entre los años 2000 y 2006, el HE1 muestra una tendencia a la disminución, lo que estaría revelando que la estructura productiva de los años recientes, no está siendo capaz de generar la cantidad de empleo necesario para absorber a toda la mano de obra disponible de la economía, por lo que más de un tercio de la población ocupada se encuentra laborando bajo la figura del subempleo.

\section{Gráfico No 2.7}

Indicador de heterogeneidad estructural (EH1) medido a través del nivel de ocupación y subocupación

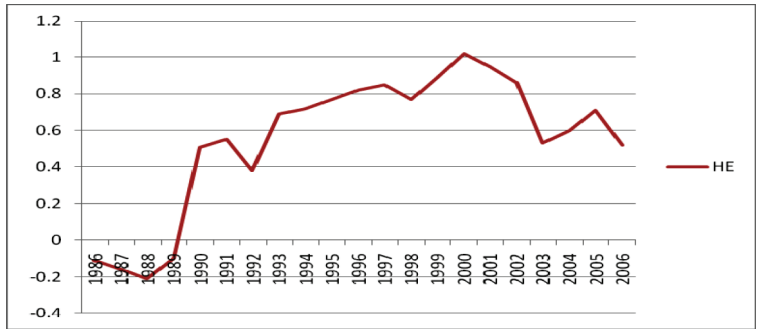

Fuente: Elaboración propia en base a datos de la EHPM, varios años. 
Los resultados nos indican entonces que una manifestación clara de la desigualdad del sistema económico salvadoreño es la existencia amplia y sistemática del subempleo al interior de la estructura ocupacional, lo cual nos confirma la presencia de Heterogeneidad Estructural $(\mathrm{HE})$, fenómeno que, a su vez, eleva las desigualdades entre los sectores productivos.
Para respaldar la elección del índice, se presenta en forma gráfica la evolución del subempleo y de los ocupados plenos en El Salvador. En el gráfico se nota que la tendencia con la que se desarrollan los ocupados plenos es mayor al desarrollo del subempleo. Pero, al mismo tiempo, se evidencia la tendencia al alza en el subempleo.

\section{Gráfico № 2.8}

Relación de los subocupados y ocupados plenos en El Salvador de los años 1986 al 2006

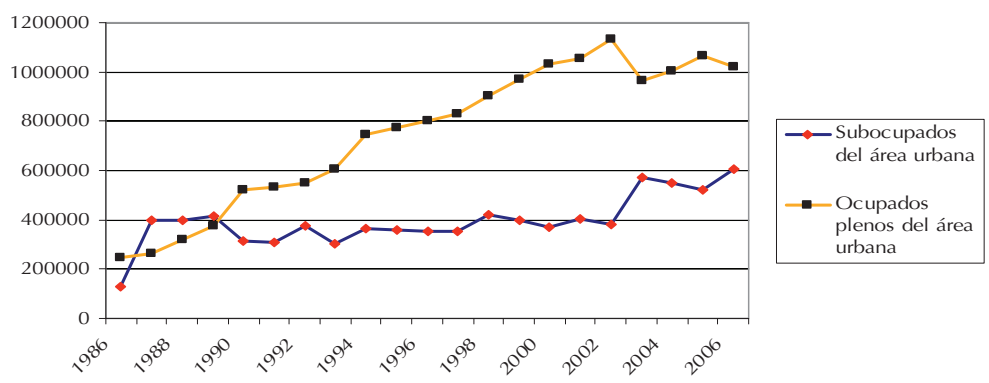

Fuente: Elaboración propia en base a datos de la EHPM, varios años.

Lo que concluimos, a partir de los gráficos anteriores, es que el mercado laboral salvadoreño, está caracterizado por una elevada participación del subempleo (propio de una economía subdesarrollada), que impacta de forma negativa sobre el aparato productivo y, de nuevo, sobre la dinámica ocupacional.

1.3.2.2. La heterogeneidad estructural medida a través de la relación del sector formal con el sector informal de la economía

$$
H E_{2}=L N\left(\frac{\text { OcupadosFormales }}{\text { OcupadosInformales }}\right) \begin{aligned}
& \mathrm{HE}>0 \mathrm{Si} \text { Ocupados Formales es mayor a Ocupados Informales. } \\
& \mathrm{HE}=0 \mathrm{Si} \text { Ocupados Formales es igual a Ocupados Informales. } \\
& \mathrm{HE}<0 \mathrm{Si} \text { Ocupados Formales es Menor a Ocupados Informales. }
\end{aligned}
$$

Este segundo indicador de heterogeneidad relaciona la población ocupada en el sector formal con la población ocupada en el sector informal de la economía. Por un lado, el sector formal, se caracteriza por su mayor dinamismo, por su uso intensivo de capital, y por sus remuneraciones relativamente altas. Por otro lado, el sector informal, se 
caracteriza por tener remuneraciones en su mayoría de subsistencia, un bajo uso de capital y por una existencia generada por la incapacidad del aparato productivo para satisfacer las aspiraciones de desarrollo y la necesidad de empleo de toda su fuerza laboral.
A continuación se establece de manera gráfica se presenta el índice de formalidad obtenido de la relación de los ocupados formales y ocupados en el sector informal.

Gráfico No 2.9

Indicador de heterogeneidad estructural (EH2) medido a través del nivel de ocupados formales y ocupados informales.

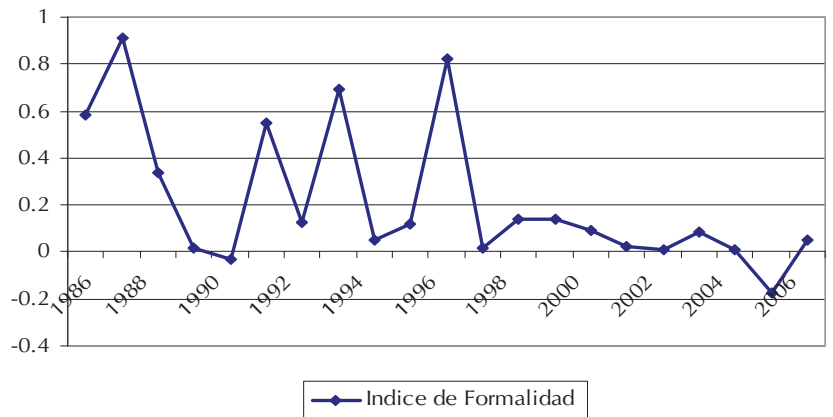

El gráfico anterior nos muestra una tendencia irregular en los datos sobre ambos sectores, por lo cual se hace necesario profundizar y presentar de forma gráfica la evolu-

\section{Gráfico No 2.10}

Relación de las tasa de crecimiento del ocupados formales e informales en El Salvador de los años 1986 al 2006.

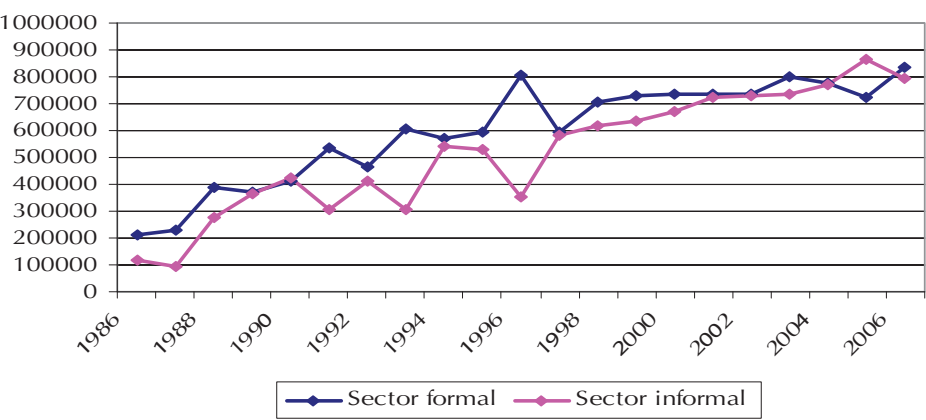

Fuente: Elaboración propia en base a datos de la EHPM varios años. ción tanto del sector de ocupados formales, como de ocupados informales, tal y como se presenta a continuación: 
La relación presentada en el gráfico anterior comprende la evolución del número de ocupados formales con respecto a los informales para el periodo de 1986-2006. Lo que debería de existir es una tendencia al incremento del número de empleados formales $y$, de manera conjunta, la reducción año con año del número de ocupados en el sector informal. No obstante, los datos oficiales nos muestran que esto no ocurre en la economía salvadoreña, por el contrario, se puede afirmar que la heterogeneidad estructural ha estado presente durante mucho tiempo. Ello ha generado el fenómeno de dualización económicosocial, que consiste en la existencia de trabajadores informales originados por la segmentación de los mercados de trabajo.

\subsubsection{La heterogeneidad estruc- tural medida a través de la activi- dad económica entre sectores.}

$$
H_{3}=L N\left(\frac{\text { IVAEterciario }}{\text { IVAEprimario }}\right) \quad \begin{aligned}
& \mathrm{HE}<0 \text { Si IVAE terciario es menor a IVAE primario. } \\
& \mathrm{HE}=0 \mathrm{Si} \text { IVAE terciario es igual a IVAE primario } \\
& \mathrm{HE}>0 \text { Si IVAE terciario es mayor a IVAE primario. }
\end{aligned}
$$

Este indicador nos muestra un cociente entre el índice de la actividad económica del sector terciario con respecto al índice de la actividad económica del sector primario. El resultado de esta división nos describe cual de los dos sectores está creciendo con mayor dinamismo. Si por ejemplo, el resultado nos muestra un número mayor que 0 , significa que existe un mayor crecimiento del sector terciario. Esto equivaldría a decir que el aparato productivo está siendo mucho más dinámico en la parte de comercio y servicios. Y si por el contrario, el cociente posee un signo negativo, equivaldría a decir que el sector primario está siendo mucho más dinámico en comparación al sector de servicios.

El resultado de este indicador ayuda a visualizar el comportamiento de la economía salvadoreña, caracterizada dar mayor importancia y apoyo al sector terciario, estructurado bajo conceptos elitistas (en los cuales se basa en el uso intensivo en capital) y del cual se puede deducir mayores niveles de desigualdad de los ingresos, reflejando la presencia de heterogeneidad estructural entre los principales sectores de la actividad económica.

\section{Análisis empírico sobre} las principales causas del problema de la desigualdad de ingresos y la exclusión social en El Salvador

La construcción de un modelo económico respecto a la desigualdad del ingreso en El Salvador, lleva consigo la tarea de establecer relaciones entre las variables que alteran o modifican el complejo comportamiento distributivo de la 
economía. Es decir, que no sólo se concibe el bajo crecimiento económico como resultado de la desigualdad de los ingresos, sino también que la estructura productiva salvadoreña, dada su heterogeneidad es una de las causantes de la mala asignación de los frutos de la actividad económica.

Partiendo de esta idea, y considerando la posibilidad y la utilidad del uso del análisis cuantitativo en la sustentación empírica de la teoría que antecede a este capítulo, se han desarrollado las relaciones, haciendo uso de métodos econométricos, que en definitiva pudieran explicar la dinámica distributiva de la economía salvadoreña.

En el modelo estimado se ha considerado en primer lugar, analizar cómo el nivel de crecimiento de la economía (explicado por el Producto Interno Bruto) es influido por los incrementos en los niveles de concentración del ingreso, el cual es medido a través del índice de desigualdad GINI. Del mismo modo, se ha considerado también explicar cómo la desigual distribución de los ingresos es afectada de manera paralela por la estructura productiva salvadoreña, es decir, que se deja de razonar el problema, como sospecha de una relación causal, sino, más bien, como un mecanismo circular, que se afecta de manera alterna y sistemática, generándose así un circulo vicioso.

Por lo tanto, se busca comprobar cómo la desigualdad se mantie- ne a pesar de la existencia de políticas sociales, que, giradas desde el estado en apoyo al desarrollo de los sectores más vulnerables de la economía (Por ejemplo, el programa Red Solidaria, el FOSALUD, etc.), no son capaces de obtener el nivel de desarrollo deseado, debido a la ineficiencia existente en cuanto a la distribución de los ingresos y de la estructura productiva.

El modelo que se presenta a continuación está construido con técnicas de regresión lineal, utilizando series de tiempo para el período de 1986 al 2006. En dicho modelo, se analiza la incidencia de la concentración del ingreso, medida a través del índice Gini ${ }^{6}$, sobre el crecimiento económico, medido por el Producto Interno Bruto (PIB) a precios constantes. Asimismo, se estudia la relación entre la estructura productiva (a través del análisis de la heterogeneidad estructural), con respecto a la desigualdad de los ingresos (medida también a través del índice de Ginil). Se ha utilizado el paquete econométrico Eviews 5.1, realizándose todas las pruebas estadísticas con un intervalo de confianza del 95\%.

\subsection{Relación entre el Producto Interno Bruto y la desigualdad del ingreso}

\subsubsection{Crecimiento económico e ín- dice de Gini, el caso de El Salvador}

Se estudia la relación, por medio de regresiones lineales, del crecimiento económico salvadoreño, medido a través de su prin- 
cipal indicador macroeconómico, el Producto Interno Bruto (PIB) a precios constantes, con respecto a la desigualdad y concentración del ingreso, medida a través del índice de Gini, para un período comprendido entre los años 1985 y 2006.

Se trata de comprobar la hipótesis sobre si, para el caso salvadoreño, la desigualdad de los ingresos causa, en efecto, un impacto negativo sobre el crecimiento económico.

Para dicha comprobación, se ha utilizado como metodología estadística el método de Mínimos Cuadrados Ordinarios (MCO) y procesos autoregresivos integrados de media móvil (ARIMA). Además, cabe mencionar que las variables PIB y GINI se transformaron con logaritmos naturales, esto para hacer posible la lectura de los coeficientes directamente como elasticidades o tasas de cambio. Asimismo, la variable GINI se ha transformado con primeras diferencias (para evitar coeficientes espurios).

\subsubsection{Definición de las variables}

LNPIB = Logaritmo natural del PIB a precios constantes de 1990

LNGINI (t-1) = Logaritmo natural del índice de Gini con un año de rezago

El modelo queda establecido por la siguiente ecuación:

$$
\operatorname{LNPIB}=C+\beta 1 \operatorname{LNGINI}(t-1)+\mu
$$

De está manera se nos muestra un modelo lineal, de orden 1 , en donde el parámetro $\beta_{1}$, es el coeficiente de regresión parcial y mide la elasticidad parcial del PIB con respecto al índice de Gini, en otras palabras, mide el cambio porcentual en el PIB debido a la variación del $1 \%$ en el GINI, manteniendo todo lo demás constante.

De acuerdo a la hipótesis planteada, se espera que el signo del coeficiente de regresión parcial sea negativo

\subsection{Resultados del modelo}

Los resultados obtenidos son los siguientes:

$$
\begin{aligned}
& L N P I B=8.18776080-4.057236 L N G I N I(t-1)+\mu \\
& (31.80)^{7} \quad(-10.10)
\end{aligned}
$$


Cuadro No 3.1

Resultados estadísticos de la relación LNGINI con respecto a LNPIB.

\begin{tabular}{|c|c|c|c|c|}
\hline \multicolumn{5}{|c|}{ Variable Dependiente: LNPIB } \\
\hline \multicolumn{5}{|c|}{ Método: Mínimos Cuadrados } \\
\hline \multicolumn{5}{|c|}{ Serie (ajustada): 19862006} \\
\hline \multicolumn{5}{|c|}{ Observaciones incluidas: 21} \\
\hline Variable & Coeficiente & $\begin{array}{c}\text { Error } \\
\text { Estándar }\end{array}$ & Estadístico t & Prob. \\
\hline LNGINI(-1) & -4.057236 & 0.401673 & -10.10085 & 0.0000 \\
\hline $\mathrm{C}$ & 8.187608 & 0.257461 & 31.80135 & 0.0000 \\
\hline R-cuadrado & 0.843010 & \multicolumn{2}{|c|}{ Mean dependent var } & 10.77916 \\
\hline R-cuadrado ajustado & 0.834748 & \multicolumn{2}{|c|}{ S.D. dependent var } & 0.241501 \\
\hline S.E. of regression & 0.098173 & \multicolumn{2}{|c|}{ Criterio de Akaike } & -1.713776 \\
\hline Sum squared resid & 0.183121 & \multicolumn{2}{|c|}{ Criterio de Schwarz } & -1.614297 \\
\hline \begin{tabular}{|l|} 
Log likelihood \\
\end{tabular} & 19.99464 & \multicolumn{2}{|c|}{ F-estadístico } & 102.0272 \\
\hline Durbin-Watson stat & 1.792597 & \multicolumn{2}{|c|}{ Prob(F-statistic) } & 0.000000 \\
\hline
\end{tabular}

Fuente: Elaboración propia en base a datos de CEPAL y EHMP varios años.

El modelo nos presenta una elevada bondad de ajuste, así como parámetros estadísticamente significativos. El valor del coeficiente de la variable independiente (LNGINI(t-1)) es elástico, lo que significa que, por cada $1 \%$ de aumento en el índice de Gini con un año de rezago, la tasa de crecimiento del PIB disminuirá en aproximadamente $4 \%$, lo que permite establecer que existe una correspondencia inversa entre las variables. Si la desigualdad aumenta, el PIB verá limitado su crecimiento, y viceversa. Como se había dicho antes, la magnitud del coeficiente puede ser leído como una elasticidad parcial: La variable LNPIB es sensible a los cambios que experimenta la variable $\mathrm{LNGINI}$, por lo tanto, los aumentos o disminuciones del PIB están afectados por las modifica- ciones que presenten los niveles de desigualdad en el país.

\subsection{Conclusiones del mo- delo que relaciona PIB e índice de GINI.}

Se examinó la evolución del crecimiento económico a partir de las fluctuaciones en el nivel de concentración de la riqueza. Los resultados del modelo que vincula al PIB como variable dependiente del índice de GINI, brindó deducciones acorde a lo esperado.

Así, la relación entre el nivel del Producto Interno Bruto y el índice de GINI, resultó de signo negativo. Es decir, que acorde con la teoría, el análisis de los datos parece confirmar que existe una relación inversa entre aumentos consecutivos en la desigualdad y el crecimiento de la economía en su conjunto. 
Un resultado a remarcar es que se observa que el principal obstáculo que se interpone al éxito de los esfuerzos en la reducción de la pobreza mediante el crecimiento económico radica en la imposibilidad de los gobiernos a la aplicación de políticas que logren redistribuir los recursos y producir así una mayor rentabilidad social y, como consecuencia, un mayor nivel de crecimiento de la economía.

\subsection{Relación entre el nivel de des- igualdad del ingreso y la estructura productiva}

Además de demostrar que la desigualdad de los ingresos afecta negativamente la estructura productiva - a través de un impacto negativo de esta variable sobre el PIB-, esta sección busca explicar cómo la estructura productiva impacta a la distribución de los ingresos. Para esto, se ha logrado construir un modelo econométrico que plasma la relación previamente descrita, considerando el período que va de 1985 al 2006.

En dicho modelo se trata de comprobar la hipótesis sobre si la heterogénea estructura productiva que existe en El Salvador, afecta negativamente a la distribución de los ingresos. De resultar verdadera, indicaría que el fenómeno de la desigualdad en el ingreso pudiera tener raíces estructurales.

Para dicha comprobación, se ha utilizado metodología estadística utilizando series temporales. Asimismo se utilizó el método de Mínimos Cuadrados Ordinarios (MCO) y de procesos autorregresivos integrados de media móvil (ARIMA).

\subsubsection{Definición de las variables}

LNGINI = Logaritmo natural del índice de Gini.

LNINDICEOP = Logaritmo natural del índice de ocupados plenos/subocupados $\left(\mathrm{H}_{1}\right)$

LNFORMALIDAD = Logaritmo natural del índice de ocupados formales/informales $\left(\mathrm{H}_{2}\right)$

LNIVAE $=$ Logaritmo natural del cociente entre el IVAE del sector terciario entre el IVAE del sector primario $\left(\mathrm{H}_{3}\right)$

Para lo cual, el modelo econométrico, queda expresado por medio de la siguiente ecuación:

$$
L N G I N I=C+\beta_{l} L N I N D I C E O P+\beta_{2} L N F O R M A L I D A D+\beta_{3} L N I V A E+u
$$

Se trata de un modelo lineal de orden 1 , en donde el parámetro $\beta_{1}$ es el coeficiente de regresión parcial que mide la elasticidad parcial del GINI con respecto al índice de ocupados plenos/subocupados; $\beta_{2}$ mide la elasticidad parcial del GINI con respecto al índice de ocupados

formales/informales; y por último $\beta_{3}$ mide la elasticidad parcial del GINI con respecto al índice del IVAE del sector terciario entre el IVAE del sector primario. En otras palabras, se busca medir las variaciones porcentuales en el GINI debido a variaciones porcentuales en las variables 
independientes, manteniendo todo

lo demás constante.

De acuerdo con la hipótesis planteada, se espera que los signos de los coeficientes de la regresión parcial sean:

$$
L N G I N I=C+\beta_{1} L N I N D I C E O P+\beta_{2} L N F O R M A L I D A D+\beta_{3} L N I V A E+u
$$$$
<0
$$

\subsubsection{Resultados del modelo}

Los resultados de la regresión son: $L N G I N I=0.65467-0.01961 L N F O R M A L-0.09562 L N I N D I C E O P-0.02725 L N I V A E+\mu$
(1.15)
$(-0.72)$
$(-2.19)$
$(-0.25)$

\section{Cuadro $\mathrm{N}^{\circ} 3.2$}

Resultados estadísticos de la relación LNINDICEOP, LNFORMAL y LNIVAE con respecto a LNGINI.

\begin{tabular}{|c|c|c|c|c|}
\hline \multicolumn{5}{|c|}{ Variable dependiente: LNGINI } \\
\hline \multicolumn{5}{|c|}{ Método: Mínimos Cuadrados } \\
\hline \multicolumn{5}{|c|}{ Serie (ajustada): 19862006} \\
\hline \multicolumn{5}{|c|}{ Observaciones incluidas: 20 después de ajustadas } \\
\hline Variable & Coeficiente & $\begin{array}{c}\text { Error } \\
\text { Estándar }\end{array}$ & Estadístico t & Prob. \\
\hline LNFORMALIDAD & -0.019610 & 0.027188 & -0.721285 & 0.4805 \\
\hline LNINDICEOP & -0.095620 & 0.043545 & -2.195918 & 0.0423 \\
\hline LNIVAE & -0.027255 & 0.107290 & -0.254032 & 0.8025 \\
\hline $\mathrm{C}$ & 0.654678 & 0.566063 & 1.156547 & 0.2635 \\
\hline R-squared & 0.670779 & \multicolumn{2}{|c|}{ Mean dependent var } & -0.642329 \\
\hline Adjusted R-squared & 0.612681 & \multicolumn{2}{|c|}{ S.D. dependent var } & 0.050489 \\
\hline S.E. of regression & 0.031422 & \multicolumn{2}{|c|}{ Akaike info criterion } & -3.912986 \\
\hline Sum squared resid & 0.016785 & \multicolumn{2}{|c|}{ Schwarz criterion } & -3.714029 \\
\hline Log likelihood & 45.08635 & \multicolumn{2}{|c|}{ F-statistic } & 11.54568 \\
\hline Durbin-Watson stat & 1.836950 & \multicolumn{2}{|c|}{ Prob(F-statistic) } & 0.000228 \\
\hline
\end{tabular}

Fuente: Elaboración propia en base a datos de CEPAL y EHMP varios años.

La ecuación nos presenta la relación que tiene la variable dependiente (LNGINI), con las variables independientes (LNFORMALIDAD, LNINDICEOP y LNIVAE). Para este caso se dice que si el índice de formalidad (LNFORMALIDAD) crece en una unidad porcentual la desigualdad medida a través del índice de Gini disminuye en $0.01961 \%$ y si el índice de ocupados plenos (LNINDICEOP) aumenta en una unidad porcentual la desigualdad se disminuiría en $0.09562 \%$ y en el 
caso del índice del IVAE (LNIVAE) si este crece en una unidad porcentual la desigualdad disminuirá en $0.02725 \%$.

Por lo tanto, vemos como las variables LNINDICEOP y LNFORMALIDAD, logran explicar el fenómeno de heterogeneidad estructural, ya que sus signos concuerdan con las hipótesis formuladas sobre el impacto de la estructura productiva sobre la desigualdad. Para el caso, el valor de la variable LNINDICEOP (resultado del cociente: ocupados plenos/subempleados) disminuirá la desigualdad a medida que la brecha entre el nivel de ocupados plenos y subempleados, se incline a favor de los primeros.

Es importante resaltar que la selección de la variable LNINDICEOP como interpretativa de heterogeneidad estructural, obedece a que con el paso del tiempo, el número de subempleados ha ido creciendo, producto principalmente del nivel de desempleo del sector primario y secundario de la economía, lo que pone de manifiesto que el aparato productivo actual (dinamizado por el auge en el sector terciario) no logra generar la cantidad de empleo suficiente para absorber a la población económicamente activa, la cual recurre principalmente a subemplearse para suplir sus necesidades básicas.

De la misma manera, el índice de formalidad (el cual resulta del cociente ocupados en el sector formal / ocupados en el sector informal), nos ayuda a poner en evidencia la presencia de heterogeneidad estructural, debido al rápido crecimiento que se refleja en el sector informal de la economía, producto principalmente al desempleo en las actividades primarias de la economía.

Sin embargo, dentro del modelo econométrico propuesto, se observa que tanto la variable LNFORMALIDAD, como la variable LNIVAE, no presentan evidencia estadística suficiente para apoyar las hipótesis esperadas. La variable LNFORMALIDAD presenta un valor estadístico no significativo, mientras que la variable LNIVAE nos presenta un signo opuesto al esperado.

De lo anterior se intuye que, en primer lugar, pudiera existir un problema de multicolinealidad, debido a que las variables regresoras se encuentran fuertemente interrelacionadas. $Y$ dado que un supuesto estadístico básico es que las variables explicativas deben ser linealmente independientes, se ha considerado elaborar un modelo de regresión simple para cada una de las variables, utilizando la variable LNGINI como dependiente en los todos los casos. Y si las variables que no dan significativas, es decir, LNFORMALIDAD y LNIVAE, pasan a ser significativas, es un indicio de que el modelo recién presentado adolece de multicolinealidad. 
2.2.2. Relación entre el índice de Gini y el índice de ocupación

En primer lugar, se elaborará un modelo de regresión que compare el índice de concentración del ingreso GINI, con el índice de ocupados plenos/subempleados.

Para lo cual el modelo econométrico queda enunciado de la siguiente manera:

$$
L N G I N I=C+\beta L N I N D I C E O P+\mu 3.5)
$$

Se trata de un modelo lineal de primer orden, del cual se espera que un signo negativo explique que la mayor ocupación plena (mantede subempleados), logre disminuir la desigualdad de los ingresos en El Salvador. A continuación se presentan los resultados obtenidos:

niendo o diminuyendo el número

Cuadro No 3.3

Resultados estadísticos de la relación LNINIDCEOP con respecto a LNGINI.

\begin{tabular}{|c|c|c|c|c|}
\hline \multicolumn{5}{|c|}{ Variable Dependiente: LNGINI } \\
\hline \multicolumn{5}{|c|}{ Método: Mínimos Cuadrados } \\
\hline \multicolumn{5}{|c|}{ Serie (ajustada): 19862006} \\
\hline \multicolumn{5}{|c|}{ Observaciones incluidas: 21} \\
\hline Variable & Coeficiente & $\begin{array}{c}\text { Error } \\
\text { Estándar }\end{array}$ & Estadístico t & Prob. \\
\hline LNINDICEOP & -0.095189 & 0.019644 & -4.845605 & 0.0001 \\
\hline $\mathrm{C}$ & -0.590605 & 0.012571 & -46.98039 & 0.0000 \\
\hline R-squared & 0.540017 & \multicolumn{2}{|c|}{ Mean dependent var } & -0.637893 \\
\hline Adjusted R-squared & 0.517018 & \multicolumn{2}{|c|}{ S.D. dependent var } & 0.053486 \\
\hline S.E. of regression & 0.037171 & \multicolumn{2}{|c|}{ Akaike info criterion } & -3.660060 \\
\hline Sum squared resid & 0.027634 & \multicolumn{2}{|c|}{ Schwarz criterion } & -3.560874 \\
\hline Log likelihood & 42.26066 & \multicolumn{2}{|c|}{ F-statistic } & 23.47989 \\
\hline Durbin-Watson stat & 1.506481 & \multicolumn{2}{|c|}{ Prob(F-statistic) } & 0.000098 \\
\hline
\end{tabular}

Fuente: Elaboración propia en base a datos de CEPAL y EHMP varios años.

Del cuadro anterior, se obtuvo la ecuación básica del modelo, tal y como se explica a continuación:

$$
\begin{aligned}
L N G I N I=- & 0.590605-0.095189 L N I N D I C E O P+\mu \\
& (-46.98)
\end{aligned}
$$

Podemos corroborar que la relación de signos entre la variable independiente y la dependiente se mantuvo: al aumentar el índice de ocupados plenos en una unidad

porcentual, la desigualdad disminuye en -0.095189 , y viceversa.

La magnitud del coeficiente $\beta$ puede ser leído como elasticidad parcial, ya que se puede observar 
que la variable LNGINI es sensible a los cambios que experimente la variable LNINDICEOP, por lo tanto los aumentos o disminuciones porcentuales del GINI están afectados por las modificaciones porcentuales que presente el índice de ocupación plena.

Siguiendo con el análisis de la regresión planteada, es necesario verificar la significancia estadística del modelo, para lo cual, se plantean las siguientes hipótesis:

$H_{o}: B_{1}=0$, El parámetro del modelo es igual a 0

$$
H_{1}: B_{1} \neq 0 \text {, El parámetro del }
$$
modelo es diferente de 0

Se cuenta con 19 grados de libertad y con un nivel de significancia del $95 \%$, el t de tablas tiene un valor de 2.093 y -2.093. Por lo tanto, si el valor t encontrado mediante la regresión tiene un valor de -4.845605 , se tiene evidencia suficiente para rechazar la hipótesis nula y estar a favor de la hipótesis alternativa, que explica que el modelo tiene significancia estadística.

\subsubsection{Relación entre el índice de} Gini y el índice de formalidad

En este caso, se ha elaborado un modelo de regresión que compara el índice de concentración del ingreso Gini, con respecto al índice de ocupados en el sector formal/ ocupados del sector informal.

El modelo econométrico queda enunciado de la siguiente manera:

Al igual que en el caso anterior, estamos en presencia de un modelo lineal de primer orden, del cual se espera que un signo negativo explique que una mayor ocupación formal (manteniendo o diminuyendo el número de ocupados informales) logre disminuir la desigualdad de los ingresos en El Salvador.

A continuación se presentan los resultados obtenidos: 
Cuadro No 3.4

\section{Resultados estadísticos de la relación LNFORMALIDAD con respecto a LNGINI}

\begin{tabular}{|c|c|c|c|c|}
\hline \multicolumn{5}{|c|}{ Variable Dependiente: LNGINI } \\
\hline \multicolumn{5}{|c|}{ Método: Mínimos Cuadrados } \\
\hline \multicolumn{5}{|c|}{ Serie (ajustada): 19872006} \\
\hline \multicolumn{5}{|c|}{ Observaciones incluidas: 20} \\
\hline Variable & Coeficiente & $\begin{array}{c}\text { Error } \\
\text { Estándar }\end{array}$ & Estadístico t & Prob. \\
\hline LNFORMALIDAD & 0.057060 & 0.035578 & 1.603801 & 0.1253 \\
\hline $\mathrm{C}$ & -0.654704 & 0.013118 & -49.90937 & 0.0000 \\
\hline R-squared & 0.119236 & \multicolumn{2}{|c|}{ Mean dependent var } & -0.642329 \\
\hline Adjusted R-squared & 0.072880 & \multicolumn{2}{|c|}{ S.D. dependent var } & 0.050489 \\
\hline S.E. of regression & 0.048614 & \multicolumn{2}{|c|}{ Akaike info criterion } & -3.119401 \\
\hline Sum squared resid & 0.044904 & \multicolumn{2}{|c|}{ Schwarz criterion } & -3.019923 \\
\hline Log likelihood & 34.75371 & \multicolumn{2}{|l|}{ F-statistic } & 2.572179 \\
\hline Durbin-Watson stat & 1.145719 & \multicolumn{2}{|c|}{ Prob(F-statistic) } & 0.125252 \\
\hline
\end{tabular}

Fuente: Elaboración propia en base a datos de CEPAL y EHMP varios años.

De la que se logro obtener la ecuación básica siguiente:

$$
\text { LNGINI }=-0.654704+0.057060 \text { LNFORMALIDAD }+\mu
$$

Podemos corroborar que la relación de signos entre la variable independiente con la dependiente se vio modificada, por lo cual el resultado obtenido no se corresponde con la hipótesis planteada, porque significaría que, al aumentar el índice de formalidad en una unidad porcentual, la desigualdad de aumentaría en 0.057060, y viceversa.

\subsection{Relación entre el índice de GINI y el índice de IVAE}

Por último, se ha intentado elaborar un modelo de regresión que compara el índice de concentración del ingreso GINI, con respecto promedio del Índice de Actividad Eco- nómica (IVAE) del sector terciario entre el sector primario.

Para lo cual el modelo econométrico queda enunciado de la siguiente manera:

$$
L N G I N I=C+\alpha L N I V A E+\mu(3.7)
$$

Del presente modelo, se espera que un signo positivo explique una relación directa entre el auge de la tercerización de la economía y las mayores desigualdades de los ingresos en El Salvador. A continuación, se presentan los resultados obtenidos: 
Cuadro No 3.5

Resultados estadísticos de la relación LNIVAE con respecto a LNGINI

\begin{tabular}{|c|c|c|c|c|}
\hline \multicolumn{5}{|c|}{ Dependent Variable: LNGINI } \\
\hline \multicolumn{5}{|l|}{ Method: Least Squares } \\
\hline \multicolumn{5}{|l|}{ Sample: 19862006} \\
\hline \multicolumn{5}{|c|}{ Included observations: 20} \\
\hline Variable & Coeficiente & $\begin{array}{c}\text { Error } \\
\text { Estándar }\end{array}$ & Estadístico t & Prob. \\
\hline LNIVAE & -0.251522 & 0.046708 & -5.384971 & 0.0000 \\
\hline $\mathrm{C}$ & -0.582673 & 0.012684 & -45.93777 & 0.0000 \\
\hline R-squared & 0.591819 & \multicolumn{2}{|c|}{ Mean dependent var } & -0.637893 \\
\hline Adjusted R-squared & 0.571410 & \multicolumn{2}{|c|}{ S.D. dependent var } & 0.053486 \\
\hline S.E. of regression & 0.035016 & \multicolumn{2}{|c|}{ Akaike info criterion } & -3.779539 \\
\hline Sum squared resid & 0.024522 & \multicolumn{2}{|c|}{ Schwarz criterion } & -3.680353 \\
\hline Log likelihood & 43.57493 & \multicolumn{2}{|c|}{ F-statistic } & 28.99792 \\
\hline Durbin-Watson stat & 1.331230 & \multicolumn{2}{|c|}{ Prob(F-statistic) } & 0.000029 \\
\hline
\end{tabular}

Fuente: Elaboración propia en base a datos de CEPAL y EHMP varios años.De la que se logro obtener la ecuación básica siguiente:

$$
L N G I N I=-0.25122-0.582673 L N I V A E+\mu
$$

En donde podemos corroborar que la relación de signos entre la variable independiente con la dependiente se vio modificada, y por lo cual el resultado obtenido no se corresponde con la hipótesis, acorde a la teoría planteada, por lo que significaría que este índice no sería el mejor indicador para la medición de desigualdad. Más sin embargo, resulta interesante observar que la inclusión de esta variable en el modelo inicial, modifica el signo de la variable LNFORMALIDAD.

\subsubsection{Conclusiones del modelo que relaciona la heterogeneidad estruc- tural con el índice de GINI}

Se ha tratado de establecer las relaciones que permitan explicar como la desigualdad es compren- dida a partir del fenómeno de heterogeneidad estructural. Para lo cual se hace uso inicialmente, de un índice de ocupación, que explique como este fenómeno ha contribuido a los aumentos sistemáticos de la desigualdad en El Salvador, coincidiendo con el informe de desarrollo humano del PNUD (PNUD, 2007:263), el cual indica que la dinámica del subempleo es consecuencia de la desarticulación del aparato productivo y una de las principales causantes de la desigualdad.

Las raíces histórico-estructurales podrían explicarse debido a que, la estructura productiva registrada en las últimas décadas, provocó el fin del modelo agroexportador 
tradicional, que se basaba en el dinamismo de las exportaciones agrícolas a mercados internacionales $y$, al mismo tiempo, el surgimiento de un nuevo modelo basado principalmente en las actividades relacionadas con los servicios y el comercio (Segovia, A. 2005:7).

El crecimiento de este sector de la economía ha sido forjado en detrimento del sector primario y secundario de la economía, sectores que producen un mayor valor agregado y que tienen una mayor capacidad de generación de empleo, lo cual ha traído como consecuencia, la agudización y el crecimiento del sector informal de la economía, que trae como secuela un elevado ritmo de crecimiento de la población subempleada.

Es curioso que, a pesar que existe una alta correlación entre las variables subempleo y ocupación informal, solamente el índice que representa el nivel de ocupados plenos con respecto a la población subempleada nos brinda un resultado acorde a lo planteado por la teoría, no así la comparación del índice de empleados formales con respecto a los informales. Esto nos hace sospechar que podría existir alguna otra variable que no ha sido incluida y que pudiera fortalecer nuestro modelo y lograr que esta variable también resulte significativa.

Otro aspecto importante es el que indica el índice del IVAE, el cual muestra las diferencias en el volumen de actividad económica que existen entre los sectores pri- mario y terciario, lo que indica la presencia de heterogeneidad, en cuanto que existe un sector más dinámico que otro. En los últimos años, como ya se dijo, El Salvador ha experimentado profundos cambios en su estrategia de crecimiento, impulsando actualmente al sector comercio, servicios y finanzas. Si bien es cierto que estas políticas han contribuido al crecimiento de algunos sectores también a generado el detrimento de otras que se consideran mas importantes por agregar mayor valor agregado a la producción.

Sin embargo, el resultado estadístico de este índice (IVAE sector terciario vrs. IVAE sector primario) no nos brinda pruebas estadísticas acordes a la teoría planteada en nuestro modelo original, lo cual nos obliga a reflexionar sobre la posibilidad de incluir alguna otra variable en el índice, para hacerlo más robusto y significativo.

De este modo, concluimos que la mayor desagregación del aparato productivo tiene alguna responsabilidad en el nivel de concentración del ingreso en El Salvador, ya que se lograron obtener resultados positivos en la medición del índice de ocupación con respecto al índice de GINI. Logrando comprobar además que no sólo la desigualdad logra afectar de manera unilateral a la estructura productiva (a través del bajo crecimiento en el PIB), sino también que la estructura productiva (por medio de la HE) logra afectar los niveles de desigualdad de la economía salvadoreña. 


\section{Conclusiones y reflexiones finales}

"Una estrategia de crecimiento económico de largo plazo debe considerar de manera integral diversas alternativas para la reducción de la desigualdad en la distribución de la riqueza como uno de los motores del desarrollo nacional"

(Daniel E. Ortega).

En El Salvador, la desigualdad económica parece ser inmune al modelo económico que se aplique. Prueba de ello es que actualmente la idea de la economía ortodoxa que plantea que la vía del crecimiento es el arma más poderosa para la mejora de la calidad de vida de las personas, no resulta eficaz en nuestros países, debido a que este crecimiento no viene aparejado de una mejora en la distribución del ingreso y la riqueza. A esto se le suma que la gran mayoría de las políticas públicas que han sido utilizadas para abordar el problema de la distribución del ingreso no han tenido los resultados esperados.

Es muy importante la revisión teórica realizada, ya que es un elemento principal en la investigación que el lector logre identificar los principales elementos contenidos en cada una de las teorías económicas de la distribución —clásica, marxista y neoclásica-. Esto debido a la necesidad de aprender sobre las raíces teóricas de la inequitativa distribución del ingreso en El Salvador; y segundo lugar, porque se considera necesario identificar aquellos mecanismos que determinan y que están presentes en la realidad distributiva capitalista salvadoreña. Es así como la desigualdad en la distribución de los recursos vigente en el sistema capitalista salvadoreño se debe a la misma dinámica del sistema, ya sea a través de la apropiación de una proporción cada vez mayor de excedente por parte de la clase capitalista, ya sea por el resultado del incremento constante de la composición orgánica del capital, de la explotación de la clase trabajadora o de las diferencias de productividad entre las distintas ramas de la economía. Definiendo así que el aumento de las precariedades de las clases sociales que ven empeorar su situación tanto en términos absolutos como en términos relativos, se debe a la propia evolución de las sociedades capitalistas.

Se percibe además, que se puede presentar una alternativa de solución a este problema de desigualdad del ingreso, que esté íntimamente ligado a las raíces de los factores que determinan la heterogénea estructura productiva (una base exportadora débil, la falta de progreso técnico, los escasos encadenamientos productivos, entre otros) y que es en conjunto lo que perpetúa y refuerza las desigualdades e impiden que el crecimiento tenga un impacto redistributivo 
acorde a los derechos sociales. No obstante, se reconoce el carácter cíclico del problema estudiado. Es decir que no se revisa de manera unidireccional cómo la desigualdad afecta la estructura productiva (vía estancamiento económico), sino que se reconoce que, de manera reciproca, la estructura productiva (vía heterogeneidad estructural) afecta de forma negativa la distribución de los recursos productivos.

Cabe mencionar que la desigualdad de los ingresos es una causante fundamental del fenómeno de pobreza y esto se debe a que se caracteriza por ser un fenómeno que afecta la condición material de los individuos, así como también su nivel cultural y sus percepciones del mundo. En El Salvador, el escaso desarrollo material de la población se ha debido, entre otras cosas, a la rigidez de la desigual estructura de distribución de los ingresos. Según datos publicados por la Encuesta de Hogares de Propósitos Múltiples, para el año 2002 el sector más rico percibía el $38.82 \%$ del total de ingresos y el sector más pobre le correspondía un $0.77 \%$ de los ingresos. Diferencias que no sufrieron grandes modificaciones para el 2006, donde un pequeño grupo percibía la mayoría de ingresos con un valor de $34.95 \%$, es la desigualdad por tanto, la que detiene el desarrollo ya que son estos pequeños grupos los que pueden estar en espacios de toma de decisiones.

Se justifica entonces que en el país los elevados niveles de des- igualdad, son los que generan la carencia de bienes necesarios para vivir, reproduciendo el fenómeno de la pobreza, y es que ello no es un fenómeno accidental ni se da en forma homogénea. Es necesario entonces, disminuir al grupo de la población que cuenta con recursos limitados para satisfacer sus necesidades. Ya que de lo contrario, se crea un escenario propicio para la inestabilidad socio-política, y como resultado, se provoca un freno para el crecimiento de la economía.

Para conocer el desenvolvimiento de esta desigualdad, se construyeron diversos indicadores de desigualdad, entre ellos, el índice de Theil, Atkinson y Gini. Además, se ha buscado presentar una exposición alternativa para identificar la situación de la heterogénea estructura productiva salvadoreña, ya que no se desea presentar únicamente la medición de este fenómeno de manera unidireccional, sino que se busca incorporar a la investigación un nuevo indicador que demuestre la disparidad de productividad entre los sectores de la economía salvadoreña que generan desigualdad.

Dicha heterogeneidad coincide, con la precarización del empleo y la descomposición de la dinámica ocupacional, que dan origen a la segmentación del mercado laboral. Los principales hallazgos de esta investigación refuerzan que en $\mathrm{El}$ Salvador existe una relación entre la heterogeneidad estructural en el aparato productivo y la presencia 
de heterogeneidad en el aparato laboral.

Ciertamente, el estilo de desarrollo del mercado laboral obedece a la falta de encadenamientos entre los sectores productivos. Esto ha generado una expansión acelerada de la población subempleada, así como de los ocupados en el sector informal. Como consecuencia, el sector informal salvadoreño ha aumentado de modo constante a lo largo de las dos últimas décadas. El crecimiento de este sector, intensivo en mano de obra no calificada, es alarmante, ya que presenta niveles bajos de ingresos que tan solo logra cubrir el consumo inmediato.

Para comprobar la relación, tanto de la estructura productiva con respecto a la desigualdad, y viceversa, se realizaron modelos econométricos que permitieron en primer lugar, ratificar la vinculación de la desigualdad con respecto al bajo crecimiento económico. Las pruebas realizadas arrojaron que el índice de desigualdad Gini es inversamente relacionado con la evolución del Producto Interno Bruto. Ya que para el periodo estudiado (de 1986 al 2006) se obtuvieron coeficientes negativos de esta relación, lo que confirmaría que un obstáculo serio del modelo de crecimiento salvadoreño es la desigual estructura distributiva entre la población.

Asimismo y de manera conjunta, se explicó como la desarticulación de la estructura productiva salvadoreña altera los índices de desigualdad. Para realizar este cálculo se genero una relación de heterogeneidad estructural, explicada por la composición del mercado laboral salvadoreño. Las pruebas arrojaron que este índice de heterogeneidad estructural (construido por la dinámica del subempleo) y que representa la desarticulación del aparato productivo, esta inversamente relacionado con la evolución del índice de desigualdad de Gini. Ello permite reforzar la hipótesis según la cual el fenómeno de la desigualdad afecta y se ve afectado de manera paralela por la estructura productiva

De esta manera, se puede recomendar que en El Salvador se genere una dinámica de desarrollo hacia adentro, es decir, que en primer lugar se busque la dinamización de las ramas de actividad económica que generan un mayor valor agregado, puesto que actualmente la dinámica de desarrollo está enfocada hacia el exterior. De esta forma, al no encontrarse articulada con los sectores primarios de la economía, la dinámica de desarrollo genera la "tercerización económica". Este fenómeno orienta a la economía de manera sistemática al auge del sector comercio y servicios, sectores que dado su bajo valor agregado, posee niveles mínimos de absorción laboral y lo cual trae como resultado la expansión de nuevas relaciones laborales como la informalidad y el subempleo.

Además puede afirmarse que uno de los principales problemas 
de El Salvador en cuanto a la dinámica ocupacional, no son los índices de desempleo, sino más bien el crecimiento sistemático de la informalidad y del subempleo, lo que imposibilita reducir los niveles de desigualdad, y estos, a su vez, obstaculizan el crecimiento de la economía en su conjunto.

De manera particular se destaca que lo novedoso de la presente pro-

\section{Bibliografía}

Acevedo, C.; (2007) Cuadernos de Desarrollo Humano, Programa de las Naciones Unidas para el Desarrollo, Mayo 2007, $N^{\circ}$ 6, San Salvador, El Salvador.

Aguilar, L.; Parada, M.; (2007) La pobreza al interior del capitalismo: ¿Fenómeno marginal o resultado estructural del sistema económico? Tesis de Licenciatura. El Salvador, Departamento de Economía, Universidad Centroamericana José Simeón Cañas.

Alfaro, G.; Escoto, A. y Sánchez, E.; (2006) La heterogeneidad estructural y la dinámica laboral en El Salvador, Tesis de Licenciatura. El Salvador, Departamento de Economía, Universidad Centroamericana José Simeón Cañas.

Alegría, S.; Bustamante, V.; Rivera, C. y Salgado, C.; (2004) La redistribución del ingreso y su impacto en la actividad productiva nacional, Tesis de Licencia- puesta, radica en que los procesos detallados a lo largo de la presente investigación no representan soluciones coyunturales y no dependen exclusivamente del desenvolvimiento del entorno económico externo. Sino, más bien, obedecen a factores que estructuralmente afectan el dinamismo de los mercados internos y del cual se espera que sea una alternativa de discusión.

tura. El Salvador, Departamento de Economía, Universidad Centroamericana José Simeón Cañas.

Brugger, S (2007) Un intento pedagógico de enseñar las distintas teorías de la distribución. [en línea]. Disponible en: http:// samuel.brugger.googlepages. com/. (Accesado el día 8 de mayo de 2008).

CEPAL (2003) Hacia el objetivo del milenio de reducir la pobreza en América Latina y el Caribe. Santiago De Chile, CEPAL.

CEPAL (2005) La esquiva equidad en el desarrollo latinoamericano. Santiago de Chile, CEPAL.

Centro de Información, Documentación y Apoyo a la Investigación (CIDAI). (2007) " ¿Democracia Salvadoreña?", Revista Proceso, No 1240, Mayo de 2007. San Salvador, El Salvador.

David Ricardo, (1817) Principios de economía política y tributación. España. 
Dirección General de Estadísticas y Censos, (Varios años) Encuesta de Hogares y Propósitos Múltiples, San Salvador, El Salvador.

Enkerlin, E, (1997), Ciencia ambiental y desarrollo sostenible. Internacional Thompson editores. México.

Escobar, B.; Zepeda, C.; (2003) La economía solidaria como alternativa económica para El Salvador. Tesis de Licenciatura. El Salvador, Departamento de Economía, Universidad Centroamericana José Simeón Cañas.

Esquivel, G,; M. Jenkins y F. Larraín (1998) Export Processing Zones in central America, Harvard Institute for Internacional development, Cambridge.

Fundación Europea (1995) Los servicios del bienestar público y exclusión social: El desarrollo de iniciativas orientadas hacia los consumidores en la Unión Europea, Dublín.

Gacitúa, E.; Sojo C., (2000) Exclusión social y reducción de la pobreza en América Latina y el Caribe, Banco Mundial, Washington, Estados Unidos.

González, H.; Rosales, C.; (2005) Distribución del Ingreso, pobreza y necesidades de crecimiento en El Salvador. Tesis de Licenciatura. El Salvador, Departamento de Economía, Universidad Centroamericana José Simeón Cañas.
Kay, C, (1991) "Estructuralismo y teoría de la dependencia en el periodo neoliberal". Revista Nueva Sociedad, Número 158, noviembre-diciembre 1998, pp. 10-25.

Kay, C, (1991) "Teorías latinoamericanas del desarrollo". Revista Nueva Sociedad, Número 113, mayo-Junio 1991, pp. 30-45.

Koutsoyiannis, (2000) Macroeconomía. España.

Martínez, J, (2007) El pensamiento de John Stuart Mill y la decadencia de la economía política clásica. San Salvador, El Salvador.

Martínez, J, (2007) El pensamiento económico de David Ricardo y los principios de economía política y tributación 1817. San Salvador, El Salvador.

Medina, F.; (2001) Consideraciones del Índice de Gini para medir la concentración del Ingreso, Estudios estadísticos y prospectivos, CEPAL, Santiago de Chile, Chile.

Ministerio de Planificación, (2007) Distribución del Ingreso e Impacto Distributivo del gasto social, Encuesta de caracterización socioeconómica nacional. Santiago de Chile.

Montoya, A, (2000), Apuntes de desarrollo económico. Editores Críticos. San Salvador, El Salvador. 
Noklen, D,; Strum, N, (1982) "La heterogeneidad estructural como concepto básico en la teoría del desarrollo". Revista de Estudios Políticos (Nueva Época), Número 28, JulioAgosto 1982, pp. 23-50.

Parada, M.; Delgado, M.; (2004) Determinantes estructurales del salario y salarios de eficiencia. Tesis de Licenciatura. El Salvador, Departamento de Economía, Universidad Centroamericana José Simeón Cañas.

Pasinetti, $\mathrm{L}(\mathrm{s} \backslash \mathrm{f})$ Crítica de la teoría neoclásica, del crecimiento y la distribución. [en línea].Disponible en: http://www.geocities. com. (Consultado el día 20 de junio 2008).

Pérez, O y M. Tablas, (2004) Pobreza y Desigualdad en El Salvador para el quinquenio 1999-2003. Un análisis cuantitativo. Tesis de licenciatura. El Salvador, Departamento de Economía, Universidad Centroamericana José Simeón Cañas.

Piola M, (1999) La exclusión social en Argentina: Novedades y perspectivas [en línea]. Disponible en: http://www.ub.es/geocrit/sn-45-25.htm (Consultado el día 25 de junio 2008).
Programa de las Naciones Unidas para el Desarrollo, (2003) Informe sobre Desarrollo Humano, San Salvador, El Salvador.

PNUD, (2008). Informe Sobre el Desarrollo Humano El Salvador 2007-2008, San Salvador, El salvador.

Ray, D, (2002) Economía del desarrollo. Edición de Antoni Bosch. España.

Rodríguez, $\mathrm{O},(\mathrm{s} \mid \mathrm{f})$ El estructuralismo latinoamericano. México, D.F.

Rubio, S Estabilidad en el empleo en El Salvador 1994-2004. Revista Alternativa para el desarrollo, N 97 FUNDE, eneromarzo de 2006.

Sánchez, D, (2005) Inserción externa, Heterogeneidad estructural y globalización en América Latina. Londres.

Townsend, P. (2003) "La conceptualización de la pobreza". Revista Pobreza: Desarrollos conceptuales y metodológicos. Mayo de 2003, vol. 53, núm. 5 de Comercio Exterior. Julio Boltvinik, México.

Wagle, U. (2000) Volver a pensar la pobreza: Definición y mediciones, Policy Science, Massachuset, Estados Unidos. 


\section{Notas}

1 Los datos utilizados para calcular los diferentes índices de desigualdad fueron proporcionados por la División de Estadísticas Social de la DIGESTYC, para los cuales los datos proporcionados del año 2004 se repiten para el 2005

2 El índice de Gini es el valor que corresponde al área entre la curva de Lorenz y la línea de equidistribución, es esta área el valor más utilizado para medir la desigualdad, en la distribución de los ingresos. Sus valores se establecen entre $0 \mathrm{y}$ 1 (o entre 0 y 100\%), donde 0 se corresponde con la perfecta igualdad y 1 se corresponde con la perfecta desigualdad.

Su cálculo se realiza mediante la siguiente fórmula:

$$
G=\frac{1}{2 n^{2} \mu} \sum_{i=1}^{n} \sum_{j=1}^{n}\left|y_{i}-y_{j}\right|
$$

Donde:

$\mathrm{n}=$ Tamaño de la población

$\mu=$ Media de los ingresos

$\mathrm{Y}_{\mathrm{i}}=$ Ingresos

$\mathrm{G}=$ índice de Gini

3 La expresión práctica que se emplea para calcular el índice de Theil se presenta a continuación:

$$
\begin{aligned}
& T(Y, 1)=1 / n \sum_{1}^{n}\left\{Y_{i} / \mu(Y) L N\left[Y_{i} / \mu(Y)\right]\right\}, \alpha=1 \text { (1) } \\
& T(Y, 0)=1 / n \sum_{1}^{n}\left\{L N\left[\mu(Y) / Y_{i}\right]\right\}, \alpha=0
\end{aligned}
$$

Donde:

$\mathrm{N}=$ Tamaño de la población

$\mathrm{Yi}=$ Ingresos

$\varepsilon=$ Media de los ingresos

$\mathrm{LN}=$ Logaritmo natural

Donde T puede tomar un valor mínimo de 0 , que equivale a la absoluta equidad y su valor máximo es $\ln (\mathrm{n})$. Además, $\alpha$ es un parámetro que captura la sensibilidad a partes particulares de la distribución; cuando $\alpha$ toma un valor alto y positivo hace al índice más sensible a cambios en la parte alta de la distribución de los ingresos $\mathrm{y}$, cuando $\alpha$ posee un valor bajo y negativo, hace al índice más sensible en la parte baja de la distribución.

4 Este índice utiliza valores entre 0 y 1 (o entre 0 y $100 \%$ ), y hace uso de un parámetro de aversión a la desigualdad $(\varepsilon)$, de modo que, entre mayor sea el valor tomado por $(\varepsilon)$, mayor será la desigualdad en la distribución de los ingresos.

Donde:

$$
A_{\varepsilon}=1-\left[\frac{1}{n} \sum_{i=1}^{n}\left(\frac{y_{i}}{\mu}\right)^{1-\varepsilon}\right]^{\frac{1}{1-\varepsilon}}
$$

$A \varepsilon=$ Índice de Atkinson

$\mathrm{n}=$ Tamaño de la población

$\varepsilon=$ Aversión a la desigualdad

$\frac{y_{i}}{\mu}=$ Ingresos / media

5 Datos encontrados de acuerdo al método de interpolación lineal.

6 Debido a la disponibilidad de información, se ha seleccionado el indice de GINI como un indicador idoneo para el calculo de la desigualdad.

7 Los datos entre parentesis representan el valor del estadistico $\mathrm{T}$, asociado a cada una de las variables. 